\title{
Minimum-Cost QoS-Constrained Deployment and Routing Policies for Wireless Relay Networks
}

\author{
Frank Yeong-Sung Lin, ${ }^{1}$ Chiu-Han Hsiao, ${ }^{1}$ Kuo-Chung Chu, ${ }^{2}$ and Yi-Heng Liu ${ }^{2}$ \\ ${ }^{1}$ Department of Information Management, National Taiwan University, No. 1 Section 4, Roosevelt Road, Taipei 106, Taiwan \\ ${ }^{2}$ Department of Information Management, National Taipei University of Nursing \& Health Sciences, \\ No. 365, Ming Te Road, Taipei 112, Taiwan
}

Correspondence should be addressed to Chiu-Han Hsiao; chiuhanhsiao@gmail.com

Received 13 April 2013; Accepted 7 July 2013

Academic Editor: Chih-Hao Lin

Copyright (C) 2013 Frank Yeong-Sung Lin et al. This is an open access article distributed under the Creative Commons Attribution License, which permits unrestricted use, distribution, and reproduction in any medium, provided the original work is properly cited.

With the continued evolution of wireless communication technology, relaying is one of the features proposed for the 4G LTE Advanced (LTE-A) system. The aim of relaying is to enhance both coverage and capacity. The idea of relays is not new, but relaying is being considered to ensure that the optimum performance is achieved to enable the expectations or good quality of service (QoS) of the users to be met while still keeping capital expenditure (CAPEX) within the budgeted bounds of operators. In this paper, we try to stand for an operator to propose a solution that determines where and how many relays should be deployed in the planning stages to minimize the development cost. In the planning stages, we not only derive a multicast tree routing algorithm to both determine and fulfill the QoS requirements to enhance throughput, but we also utilize the Lagrangian relaxation (LR) method in conjunction with optimization-based heuristics and conduct computational experiments to evaluate the performance. Our contribution is utilizing the LR method to propose an optimal solution to minimize the CAPEX of operators to build up a relay network with more efficiency and effectiveness and the QoS can be guaranteed by service level agreement.

\section{Introduction}

Providing a guaranteed service and good performance with budgets constraint is always an optimization problem of operators and vendors. During the last decade, this problem has however become much more difficult, because the traffic has grown significantly and demand for broadband data services is expected to increase tremendously [1]. The business challenges of operators would be that increasing revenues will have to come from nonvoice services which means they have to increase total communication market shares with extending service coverage and offering good service by capacity expansion as well as increased bandwidth and improved quality of services (QoSs) [2]. But building out of the macronetworks significantly will require huge investments, especially where access would otherwise be limited or unavailable without the need for expensive cellular towers. So, the operators may plan to compensate with new revenues and cost reduction at the same time. The aim of this paper is related to the relays should be deployed strategies to investigate how the relays are suitable for providing good services by minimize operator capital expenditure (CAPEX) significantly [3].

In the ongoing standardization technology development by third-Generation Partnership Project (3GPP), relaying is one of the features proposed for the LTE Advanced (LTE-A) system [4-8]. The aim of LTE-A relaying is to enhance both coverage and capacity. However, this idea of relays is not new, but the LTE-A relaying is being considered to ensure that the optimum performance is achieved to enable the expectations of the users to be met while still keeping CAPEX within the budgeted bounds. As cell edge performance is becoming more critical, with some of the technologies being pushed towards their limits, it is necessary to look at solutions that 
will enhance performance at the cell edge for a comparatively low cost. One solution that is being investigated and proposed is that of the use of relays [9-11].

In order for the cellular telecommunications technology to be able to keep pace with technologies that may compete, it is necessary to ensure that new cellular technologies are being formulated and developed. But there are many realistic conditions influencing operators including the tough economic environment, declining budgets, limited resources, time pressures, and high user expectations.

This paper proposes a solution approach for relay network planning of where to build relays, and how to configure each relay, how the routing algorithm of relays and mobile stations is worked properly. This research can be divided into two parts. First, we constructed the relay network architecture with multicast tree routing concepts. Secondly, we proposed a precise mathematical expression to model this network and developed algorithms based on Lagrangian Relaxation Method to solve this problem. These model approaches might nevertheless be regarded as useful engineering guidelines for operators to build up a good network to extend services and reduce CAPEX efficiently and effectively.

This paper is organized as follows. Section 2 is Literature Survey, and then we introduce a mathematical formulation for the wireless relay networks design problem in Section 3. In Section 4, we present the solution approach by using Lagrangian Relaxation, in which heuristics for calculating a good primal feasible solution are developed, and conduct computational experiments. In Section 5, we conclude and discuss the direction of future research.

\section{Literature Survey}

Multihop Wireless Networking has been widely studied and implemented throughout ad hoc networks and mesh networks to exploit the user diversity concept and improve overall performance. The original concept of general relaying problems was defined in $[12,13]$ and was inspired by the development of the ALOHA system at the University of Hawaii. Based on this concept, a relay network can be designed as a tree-based topology with one end of the path being the base station (BS) relaying multiple connections to provide services and improve the coverage.

Relay stations (RSs) have some characteristics or cost efficiency for the following reasons.

(1) The transmission range is much less than a BS, meaning that the transmit power is also less than that of a BS. Relays are generally cheaper than BS, meaning reduced costs without site survey and easy to construct relays in the place which is not suitable to build a base station tower.

(2) Relays do not have a wired connection to the backhaul. Instead, they receive signals from the BS and retransmit to destination users wirelessly and vice versa. The leases of wired broadband backhauls can be saved.

(3) Relaying techniques have the dual advantages of performance improvement and coverage extension at the cell edge. These could feasibly be a deployment solution for the high-frequency band in which propagation is significantly more vulnerable to nonline-ofsight (NLOS) conditions to overcome shadowing [14].

Coordinated multipoint (CoMP) is a relatively new class of spatial diversity techniques that are enabled by relaying $[15,16]$ and cooperative communications which is shown in Figure $1[17,18]$. This concept has been the focus of many studies by 3GPP for LTE-A as well as the IEEE for the WiMAX, 802.16 standards. But still no conclusion about CoMP has been reached regarding the full implementation, because CoMP has not been included in Rel.10 of the 3GPP standards. As the work is ongoing, CoMP is likely to reach a greater level of consensus; when this occurs, it will be included in future releases of the standards.

CoMP is a complex set of techniques which are distributed radios that jointly transmit information in wireless environments. The main purpose may be improved for the reliability of communications in terms of coverage extension, reduced outage probability, symbol-error, or bit-error probability for a given transmission rate [19-21]. It brings many advantages to the user as well as the network operator as follows.

(1) It makes better utilization of network: by providing connections to several BSs or RSs at once, using CoMP, data can be passed through least loaded BS or RS for better resource utilization.

(2) It provides enhanced reception performance: using several sources cooperative BSs or RSs for each connection means that overall reception will be improved and the number of dropped calls should be reduced.

(3) Multiple site reception increases received power: the joint reception from multiple BSs or RSs using CoMP techniques enables the overall received power at the handset to be increased.

When building a relay wireless network in a metropolis, various factors influence the design such as QoS requirements, throughput requirements, and total cost. The objective of our research is "to minimize the total building costs subject to QoS and throughput requirements." Nonetheless, this objective is obviously a tradeoff because total building costs will increase if the QoS and throughput requirements increase. Based on this conventional tradeoff, we take multipath routing algorithms into consideration to solve the critical problem [22, 23].

The purpose of this research is different from that of conventional network design problems. The assumptions are that multiple source nodes jointly transmit one single source of information if the signal strength is not robust enough in the link between one source node to the destination. The routing policy is no longer a single path but a more complex and interesting multipath algorithms.

\section{Problem Formulation}

3.1. Problem Description. A sequence of the wireless relay network design may be described as fellows. First, the location 


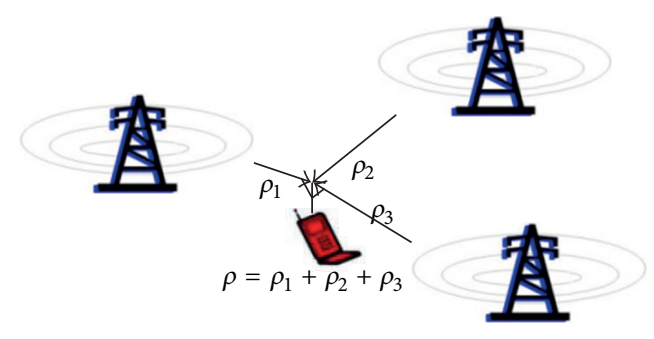

FIGURE 1: Coordinated multipoint.

of each BS could be determined by site survey and how many BSs can cover the service area. Second, the set of BSs roughly divide the entire network into several subnetworks, each of which is rooted by one BS connected to the core network which is shown in Figure 2. Meanwhile, there are many candidate locations suitable for the deployment of relays. The decision of deploying a relay at a specific location depends on the users surrounding the location; once a location is selected, the relay must associate with one of the BSs mentioned previously. So that total costs will depend on where relays are developed and how many relays should be developed of the network. But there is another important factor that should be considered by how to provide a good QoS of users at the stage of the network design. This problem may be solved by designing a mechanism or routing algorithm through which users can reach the suitable relays or BSs which can serve users well. In our work, we introduce the multicast tree-based routing algorithm (MTBR) to apply the multipath concept, as represented later.

Figure 2 illustrates the entire network design: the triangles represent the BSs; the cell phones represent the mobile stations (MSs); the circles with solid line represent the relay stations being built on the selected locations; and the circles with dotted line represent the locations not selected to build RSs. The whole area is divided into several subnetworks and rooted at associated BSs. If a subnetwork is concerned that each OD pair, like the BS-to-MS, can be expressed in Figure 3, transmits through the routing multicast tree to the associated BS. In DL transmission, data is multicasted from BS to the RSs selected by the MS, and cooperatively relayed to the destination MS to achieve the spatial diversity gains through CoMP techniques. The same routing multicast tree in UL is represented in Figure 4, in which the aggregation of traffic from the MS can overcome the weak signal strength when the MS is far from the BS or RSs. Because the channels, bandwidth, and even transmission power are different between DL and UL, the DL tree and UL tree of an MS may be different. In this paper, we derive a near optimal RSs development policy to minimize total development costs; we also maintain both DL and UL spanning trees and use the MTBR to ensure that BER and data rate requirements of each MS are satisfied.

\subsection{Mathematical Modeling}

Assumption

(i) The relaying protocol in this model is Decode-andForward.

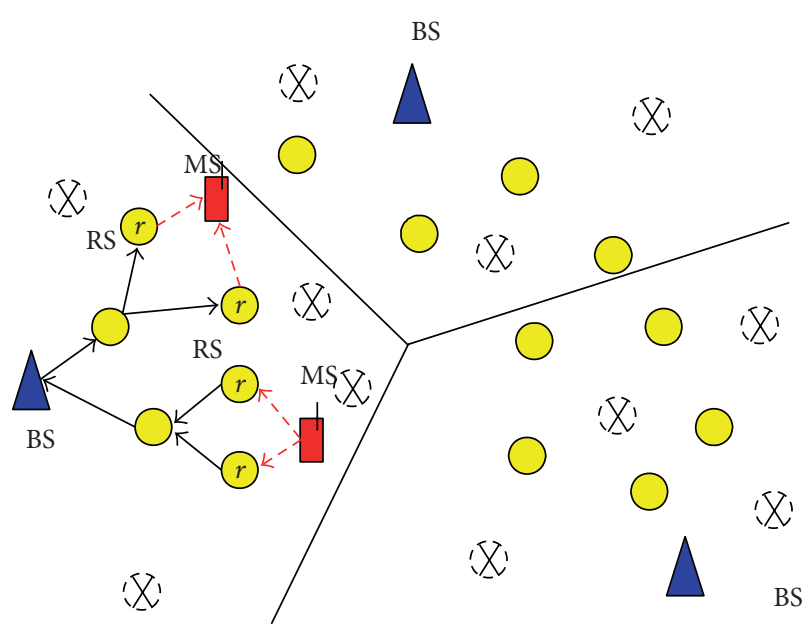

FIGURE 2: Network separations with several BSs.

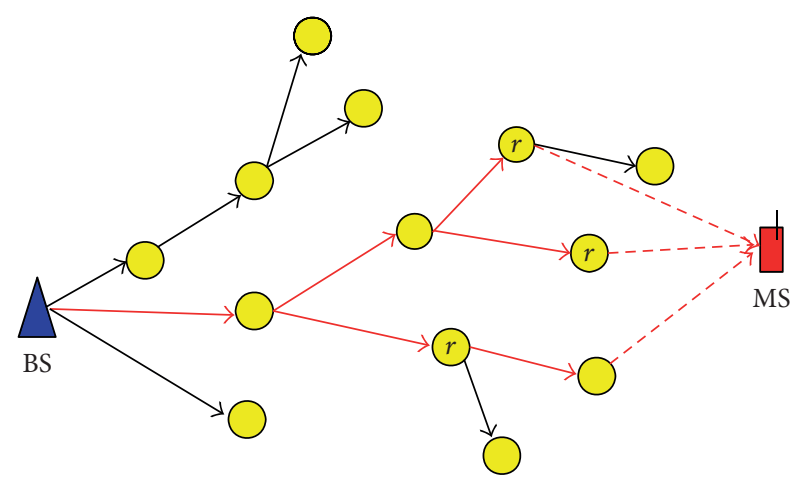

FIGURE 3: One OD pair routing multicast tree in DL transmission.

(ii) Once a location is selected to build an RS, it must home to one BS.

(iii) Each MS must home to either a BS or RS(s).

(iv) The RSs selected by an MS must associate with the same BS.

(v) The routing path of each OD pair in DL (UL) is a multicast tree.

(vi) The capacity of a link $u v$ is decided by adaptive modulation with respect to the signal-to-noise ratio (SNR) received at node $v$.

(vii) The spatial diversity gains are represented by the aggregate SNR with CoMP techniques.

(viii) The bit error rate (BER) of a transmission is measured by the receiving SNR value

(ix) The aggregate BER of the destination is the summation of BER of each node on the routing multicast tree.

(x) The numbers of links of each path adopted by each MS are assumed to be equal to ensure that the CoMP can be achieved within limited delay.

(xi) Error corrections and retransmissions are not considered in this problem. 


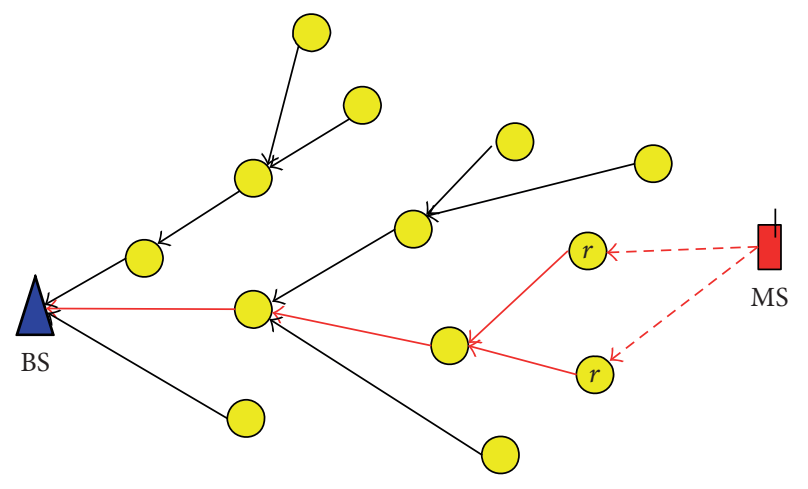

FIGURE 4: One OD pair routing multicast tree in UL transmission.

Given

(i) The set of BSs, candidate locations and configurations of RSs, MSs,

(ii) required data rate of an MS in DL and UL,

(iii) fixed and configured cost of an RS,

(iv) the set of all spanning trees, paths,

(v) distance of each link,

(vi) attenuation factor,

(vii) thermal noise function,

(viii) transmit power of BS, RS, and MS,

(ix) sNR function,

(x) the minimum SNR requirement for an $\mathrm{MS}$ in $\mathrm{DL}$ and UL to home to a BS or an RS,

(xi) the maximum BER threshold of a OD pair transmission in DL and $\mathrm{UL}$,

(xii) nodal and link capacity functions,

(xiii) the maximum spatial diversity of an MS in DL and UL.

Objective. To minimize the total cost of wireless relay network deployment.

Subject to

(i) RS selection constraints,

(ii) nodal capacity constraints,

(iii) cooperative relaying constraints in DL and UL,

(iv) routing constraints in DL and UL,

(v) link capacity constraints in DL and UL.

\section{To Determine}

(i) Whether or not a location should be selected to build an RS,

(ii) the cooperative RSs of each MS,

(iii) the routing paths of an OD pair (a BS to an MS or contrary), which form a multicast tree from the BS to the cooperative RSs selected by each MS.
Objective Function

$$
\min \sum_{r \in R} \sum_{k \in K}\left(\psi_{r}+\Phi_{r}(k)\right) \eta_{r k}
$$

Subject to

Relay Selection Constraints

$$
\sum_{k \in K} \eta_{r k} \leq 1 \quad \forall r \in R
$$

$$
\sum_{b \in B} h_{r b}^{\mathrm{dir}} \leq 1 \quad \forall r \in R, \operatorname{dir} \in \mathrm{DIR}
$$

$$
\sum_{b \in B} h_{r b}^{\mathrm{dir}} \leq \sum_{k \in K} \eta_{r k} \quad \forall r \in R, \operatorname{dir} \in \mathrm{DIR} .
$$

Nodal Capacity Constraints

$$
\begin{aligned}
& \sum_{u \in\{R \cup B\}} \sum_{n \in N} y_{n u r}^{1} \theta_{n}^{1}+\sum_{w \cup\{R \cup B\}} \sum_{n \in N} y_{n r w}^{2} \theta_{n}^{2} \\
& \leq \sum_{k \in K} \eta_{r k} \overline{C_{r}(k)} \quad \forall r \in R, \\
& \sum_{r \in R} \sum_{n \in N} y_{n b r}^{1} \theta_{n}^{1}+\sum_{i \in R} \sum_{n \in N} y_{n i b}^{2} \theta_{n}^{2} \\
& \quad+\sum_{n \in N} \sum_{\text {dir } \in \text { DIR }} \kappa_{n b}^{\operatorname{dir}} \theta_{n}^{\mathrm{dir}} \leq \overline{C_{b}} \quad \forall b \in B .
\end{aligned}
$$

Cooperative Relay Constraints

$$
\begin{gathered}
\kappa_{n r}^{\mathrm{dir}} \leq \sum_{k \in K} \eta_{r k} \quad \forall n \in N, \\
r \in R, \quad \operatorname{dir} \in \mathrm{DIR}, \\
\sum_{b \in B} \kappa_{n b}^{\mathrm{dir}}+\kappa_{n r}^{\mathrm{dir}} \leq 1 \quad \forall n \in N, \\
r \leq R, \quad \operatorname{dir} \in \mathrm{DIR}, \\
\sum_{s \in\{R \cup B\}} \kappa_{n s}^{\mathrm{dir}} \quad \forall n \in N, \operatorname{dir} \in \mathrm{DIR}, \\
\sum_{r \in R} \kappa_{n r}^{\mathrm{dir}} \leq \mathrm{SD}^{\mathrm{dir}} \quad \forall n \in N, \operatorname{dir} \in \mathrm{DIR}, \\
\kappa_{n s}^{1} P_{\mathrm{min}}^{N} \leq \kappa_{n s}^{1} \pi_{s n}^{1} \forall n \in N, s \in\{R \cup B\}, \\
\kappa_{n s}^{2} P_{\min }^{R} \leq \kappa_{n s}^{2} \pi_{n s}^{2} \quad \forall n \in N, s \in\{R \cup B\}, \\
\omega_{n} \leq \sum_{s \in\{R \cup B\}} \kappa_{n s}^{1} \pi_{s n}^{1} \quad \forall n \in N, \\
0 \leq \pi_{s n}^{\mathrm{dir}} \leq \bar{\pi}_{n} \quad \forall s \in\{R \cup B\}, \\
n \in N, \quad \operatorname{dir} \in \mathrm{DIR},
\end{gathered}
$$




$$
\begin{gathered}
\kappa_{n s}^{1} \pi_{s n}^{1} \leq \sum_{k \in K} \eta_{s k}\left[P\left(\rho_{s}^{1}(k), D_{s n}, \tau\right)-P_{N}(n)\right] \\
\forall s \in\{R \cup B\}, \quad n \in N, \\
\kappa_{n s}^{2} \pi_{n s}^{2} \leq \sum_{k \in K} \eta_{s k}\left[P\left(\rho_{n}^{N}, D_{n s}, \tau\right)-P_{N}(s)\right] \\
\forall s \in\{R \cup B\}, \quad n \in N, \\
y_{n u v}^{\mathrm{dir}} P_{\min }^{R} \leq y_{n u v}^{\mathrm{dir}} \phi_{u v}^{\mathrm{dir}} \quad \forall n \in N, \\
u, v \in\{R \cup B\}, \quad \operatorname{dir} \in \mathrm{DIR}, \\
\varepsilon_{n v} \leq \sum_{u \in\{R \cup B\}} y_{n u v}^{2} \phi_{u v}^{2} \quad \forall n \in N, \quad v \in\{R \cup B\}, \\
0 \leq \phi_{u v}^{\mathrm{dir}} \leq \bar{\phi}_{v} \quad \forall u, v \in\{R \cup B\}, \operatorname{dir} \in \mathrm{DIR}, \\
y_{n u v}^{\mathrm{dir}} \phi_{u v}^{\mathrm{dir}} \leq \sum_{k \in K} \eta_{u k}\left[P\left(\rho_{u}^{\mathrm{dir}}(k), D_{u v}, \tau\right)-P_{N}(v)\right] \\
\forall n \in N, \quad u, v \in\{R \cup B\}, \quad \operatorname{dir} \in \mathrm{DIR}, \\
\sum_{u \in\{R \cup B\}} \sum_{v \in\{R \cup B\}} y_{n u v}^{1} \mathrm{BER}\left(\phi_{u v}^{1}\right)+\mathrm{BER}\left(\omega_{n}\right) \\
\leq \mathrm{BER}{ }^{1} \quad \forall n \in N, \\
\sum_{v \in\{R \cup B\}} \operatorname{BER}\left(\varepsilon_{n v}\right)+\sum_{s \in\{R \cup B\}} \mathrm{BER}\left(\kappa_{n s}^{2} \pi_{n s}^{2}\right) \\
\leq \mathrm{BER}{ }^{2} \quad \forall n \in N .
\end{gathered}
$$

\section{Routing Constraints}

$$
\begin{aligned}
& \kappa_{n r}^{\mathrm{dir}} \leq h_{r b}^{\mathrm{dir}} \quad \forall n \in N, \\
& r \in R, \quad b \in B, \quad \operatorname{dir} \in \mathrm{DIR}, \\
& h_{r b}^{\mathrm{dir}} \leq \sum_{p \in P_{b r}} x_{n r p}^{\mathrm{dir}} \quad \forall n \in N, \\
& \quad b \in B, \quad \operatorname{dir} \in \mathrm{DIR}, \\
& \sum_{b \in B} \sum_{p \in P_{b r}} x_{n r p}^{\mathrm{dir}} \leq 1 \quad \forall n \in N, \\
& \quad r \in R, \quad \operatorname{dir} \in \mathrm{DIR}, \\
& \sum_{p \in P_{b i}} \sum_{u \in\{R \cup B\}} \sum_{v \in\{R \cup B\}} x_{n i p}^{\mathrm{dir}} \delta_{p u v} \\
& \leq \sum_{p \in P_{b j}} \sum_{u \in\{R \cup B\}} \quad \sum_{v \in\{R \cup B\}} x_{n j p}^{\mathrm{dir}} \delta_{p u v}+\left(1-\kappa_{n j}^{\mathrm{dir}}\right) \bar{M} \\
& \quad \forall n \in N, \quad i, j \in R, \quad b \in B, \quad \operatorname{dir} \in \mathrm{DIR}, \\
& y_{n u v}^{1} \leq \sum_{k \in K} \eta_{v k} \quad \forall n \in N, \\
& u \in\{R \cup B\}, \quad v \in R,
\end{aligned}
$$

$$
\begin{aligned}
& y_{n u v}^{2} \leq \sum_{k \in K} \eta_{u k} \quad \forall n \in N, \\
& u \in R, \quad v \in\{R \cup B\}, \\
& \sum_{b \in B} \sum_{p \in P_{b r}} x_{n r p}^{\mathrm{dir}} \delta_{p u v} \leq y_{n u v}^{\mathrm{dir}} \quad \forall n \in N, \\
& r \in R, \quad u, v \in\{R \cup B\}, \quad \operatorname{dir} \in \mathrm{DIR} .
\end{aligned}
$$

Link Capacity Constraints

$$
\begin{aligned}
& \sum_{n \in N} y_{u u v}^{\mathrm{dir}} \theta_{n}^{\mathrm{dir}} \leq C_{u v}\left(\phi_{u v}^{\mathrm{dir}}\right), \\
& \forall u, v \in\{R \cup B\}, \quad \operatorname{dir} \in \mathrm{DIR}
\end{aligned}
$$

Integer Constraints

$$
\begin{gathered}
\eta_{r k}=0 \text { or } 1 \quad \forall r \in R, k \in K, \\
h_{r b}^{\mathrm{dir}}=0 \text { or } 1 \quad \forall r \in R, \\
b \in B, \quad \operatorname{dir} \in \mathrm{DIR}, \\
\kappa_{n s}^{\mathrm{dir}}=0 \text { or } 1 \quad \forall n \in N, \\
s \in\{R \cup B\}, \quad \operatorname{dir} \in \mathrm{DIR}, \\
x_{n r p}^{\mathrm{dir}}=0 \text { or } 1 \quad \forall n \in N, \quad r \in R, \\
p \in P_{b r}, \quad b \in B, \quad \operatorname{dir} \in \mathrm{DIR}, \\
y_{n u v}^{\mathrm{dir}}=0 \text { or } 1 \quad \forall n \in N, \\
u, v \in\{R \cup B\}, \quad \operatorname{dir} \in \mathrm{DIR} .
\end{gathered}
$$

Explanation of Objective Function. The objective function (IP) is to minimize the total cost of RSs deployment: (1) Fix costs of RS such as land acquisition and hardware purchases; (2) The configured costs of each RS. The detail parameters in the formulation are noted and shown in Tables 1 and 2.

\section{Explanation of Constraint}

(1) Relay Assignment Constraints. Constraint (1) requires that each location is selected to install an RS with exactly only one configuration or none.

Constraint (2) requires that each RS can associate with one BS or none in direction dir.

Constraint (3) indicates that once an RS $r$ associates with a BS, $r$ must be built.

(2) Nodal Capacity Constraints. Constraint (4) requires that each RS's total amount of traffic in DL and UL cannot be greater than its nodal capacity.

Constraint (5) requires that each BS's total amount of traffic in DL and UL cannot be greater than its nodal capacity.

(3) Cooperative Relay Constraints. Each MC will select an RS $r$ in direction dir only if $r$ is installed in (6).

An MC must select either one BS or RS(s) in direction dir in (7). 
TABLE 1: Notations of given parameters.

\begin{tabular}{|c|c|}
\hline \multicolumn{2}{|r|}{ Given parameters } \\
\hline Notation & Description \\
\hline \multicolumn{2}{|l|}{ General } \\
\hline DIR & $\begin{array}{l}\text { The set of transmission direction, where } \\
\text { dir } \in \text { DIR, DIR }=\{1 \text { (downlink), } 2 \text { (uplink) }\}\end{array}$ \\
\hline$B$ & The set of BSs, where $b \in B$ \\
\hline$R$ & The set of RS candidate locations, where $r \in R$ \\
\hline K & The set of RS configurations, where $k \in K$ \\
\hline$N$ & The set of MCs, where $n \in N$ \\
\hline$\theta_{n}^{\mathrm{dir}}$ & $\begin{array}{l}\text { The data rate required to be transmitted of MC } \\
n \text { in direction dir in (packets/sec) }\end{array}$ \\
\hline$\psi_{r}$ & The fix cost of building an RS on location $r$ \\
\hline$\Phi_{r}(k)$ & $\begin{array}{l}\text { The configured cost of building RS } r \text {, which is a } \\
\text { function of configuration } k\end{array}$ \\
\hline $\bar{M}$ & An arbitrarily large number \\
\hline \multicolumn{2}{|l|}{ Routing } \\
\hline$P_{b r}$ & $\begin{array}{l}\text { The set of paths from BS } b \text { to RS } r \text {, where } \\
p \in P_{b r}\end{array}$ \\
\hline$\delta_{p u v}$ & $\begin{array}{l}\text { The indicator function which is } 1 \text { if link } u v \text { is } \\
\text { on path } p \text { and } 0 \text { otherwise }\end{array}$ \\
\hline \multicolumn{2}{|c|}{ SNR and attenuation } \\
\hline$D_{u v}$ & The distance of link $u v$ \\
\hline$\tau$ & Attenuation factor \\
\hline$\rho_{r}^{\mathrm{dir}}(k)$ & $\begin{array}{l}\text { Transmit power of RS } r \text { in direction dir, which } \\
\text { is a function of configuration } k\end{array}$ \\
\hline$P_{N}(s)$ & $\begin{array}{l}\text { Thermal noise strength function in } \mathrm{dBm} / \mathrm{Hz} \text {, } \\
\text { where } s \in\{N, R, B\} \text { represents receiving node } \\
\text { type. }\end{array}$ \\
\hline$\rho_{b}^{B}$ & Transmit power of BS $b$ \\
\hline$\rho_{n}^{N}$ & Transmit power of MC $n$ \\
\hline$P\left(\rho_{u}^{\mathrm{dir}}(k), D_{u v}, \tau\right)$ & $\begin{array}{l}\text { Signal strength received by node } v \text { in } \mathrm{dBm} \text {, } \\
\text { which is a function of } \rho_{r}^{\text {dir }}(k), D_{u v} \text { and } \tau\end{array}$ \\
\hline$P_{\min }^{N}$ & $\begin{array}{l}\text { The minimum SNR requirement for a } \mathrm{MC} \text { to } \\
\text { receive from a RS in } \mathrm{DL}\end{array}$ \\
\hline$P_{\min }^{R}$ & $\begin{array}{l}\text { The minimum SNR requirement for a RS to } \\
\text { receive from a MC in UL }\end{array}$ \\
\hline $\bar{\phi}_{v}$ & $\begin{array}{l}\text { The maximum SNR can be received by node } v \\
\text { in link } u v \text {, where } u, v \in\{R \cup B\}\end{array}$ \\
\hline $\bar{\pi}_{v}$ & $\begin{array}{l}\text { The maximum SNR can be received by node } v \\
\text { in link } u v \text {, where } u \in\{R \cup B\}, v \in N \text { in DL; and } \\
u \in N, v \in\{R \cup B\} \text { in } \mathrm{UL}\end{array}$ \\
\hline \multicolumn{2}{|l|}{ BER } \\
\hline BER $^{\text {dir }}$ & $\begin{array}{l}\text { The BER requirement for the transmission } \\
\text { received by a destination in direction dir where } \\
\text { the destination in DL is MC and in UL is BS }\end{array}$ \\
\hline $\operatorname{BER}\left(\mathrm{SNR}_{s}\right)$ & $\begin{array}{l}\text { The BER value of each node } s \text {, which is a } \\
\text { function of the receiving SNR, where } \\
s \in\{R \cup B \cup N\}\end{array}$ \\
\hline
\end{tabular}

TABle 1: Continued.

Given parameters

\begin{tabular}{ll} 
Notation & Description \\
\hline $\begin{array}{l}\overline{C_{b}} \\
\overline{C_{r}(k)}\end{array}$ & $\begin{array}{l}\text { The nodal capacity of } \mathrm{BS} b \text { in (packets/sec) } \\
\text { The nodal capacity of } \mathrm{RS} r \text { in (packets } / \mathrm{sec}), \\
\text { which is a function of configuration } k\end{array}$ \\
$C_{u v}(\mathrm{SNR})$ & $\begin{array}{l}\text { The capacity of link } u v \text { in (packets/sec), which } \\
\text { is a function of the receiving SNR of node } v, \\
\text { where } u, v \in\{R \cup B\}\end{array}$ \\
\hline Relaying & $\begin{array}{l}\text { The maximum spatial diversity of a MC in } \\
\text { direction dir }\end{array}$ \\
\hline
\end{tabular}

TABLE 2: Notations of decision variables.

\begin{tabular}{|c|c|}
\hline Notation & Description \\
\hline \multicolumn{2}{|c|}{ Decision variables } \\
\hline$\eta_{r k}$ & $\begin{array}{l}1 \text { if candidate location } r \text { is selected to build a RS } \\
\text { with configuration } k \text { and } 0 \text { otherwise }\end{array}$ \\
\hline$h_{r b}^{\mathrm{dir}}$ & $\begin{array}{l}1 \text { if RS } r \text { associates with BS } b \text { in direction dir and } 0 \\
\text { otherwise }\end{array}$ \\
\hline$\kappa_{n s}^{\mathrm{dir}}$ & $\begin{array}{l}1 \text { if node } s \text { is selected to cooperatively relay the } \\
\text { transmission of } \mathrm{MC} n \text { in direction dir and } 0 \\
\text { otherwise, where } s \in\{R \cup B\}\end{array}$ \\
\hline$y_{n u v}^{\mathrm{dir}}$ & $\begin{array}{l}1 \text { if link } u v \text { is on the multicast tree adopted by MC } \\
n \text { in direction dir and } 0 \text { otherwise }\end{array}$ \\
\hline$x_{n r p}^{\mathrm{dir}}$ & $\begin{array}{l}1 \text { if path } p \text { is selected for MC } n \text { to cooperative RS } r \\
\text { in direction dir and } 0 \text { otherwise, where } p \in P_{b r}\end{array}$ \\
\hline \multicolumn{2}{|c|}{ Auxiliary variables } \\
\hline$\phi_{u v}^{\mathrm{dir}}$ & $\begin{array}{l}\text { The SNR received by node } v \text { in link } u v \text {, where } \\
u, v \in\{R \cup B\}\end{array}$ \\
\hline$\pi_{u v}^{\mathrm{dir}}$ & $\begin{array}{l}\text { The SNR received by node } v \text { in link } u v \text {, where } \\
u \in\{R \cup B\}, v \in N \text { in DL; and } u \in N, v \in\{R \cup B\} \\
\text { in UL }\end{array}$ \\
\hline$\omega_{n}$ & The summation of SNR received by $\mathrm{MC} n$ in $\mathrm{DL}$ \\
\hline$\varepsilon_{n s}$ & $\begin{array}{l}\text { The summation of SNR received by node } s \text { in UL } \\
\text { oriented by MC } n \text {, where } s \in\{R \cup B\}\end{array}$ \\
\hline
\end{tabular}

Constraints (8) and (9) represent the boundaries of the number of cooperative RSs an MC can select.

The minimum SNR constraints for an MC to receive from a BS or an RS in DL, and for an MC to transmit to a BS or an RS in UL, are expressed in (10) and (11), respectively.

Constraint (12) requires that the SNR value received by an MC $n$ in DL cannot exceed the summation of the SNR values $n$ receives from the cooperative RSs selected by $n$.

Constraint (13) represents the boundaries of decision variable $\pi_{u v}^{\text {dir }}$.

Once MC $n$ selects RS (or BS) $s$ to be its cooperative RS, the SNR value on link $n s$ cannot exceed the SNR transmitted from source node to destination node in DL and UL in constraints (14) and (15).

The minimum SNR constraint for a link $u v$ selected by MC $n$ is expressed in (16), while $u, v \in\{R \cup B\}$. 
Constraint (17) requires that the SNR value received by an RS (or BS) $v$ in UL cannot exceed the summation of the SNR values on the link $u v$ selected by MC $n$.

Constraint (18) represents the boundaries of decision variable $\phi_{u v}^{\mathrm{dir}}$.

Once MC $n$ selects a link $u v$ in direction dir, the SNR value on $u v$ cannot exceed the SNR transmitted from $u$ to $v$ in (19).

The aggregative BERs constraints for DL in MC and UL in BS are expressed in (20) and (21), respectively.

(4) Routing Constraints. Constraint (22) requires that once RS $r$ is selected by MC $n, r$ must associate with one BS in direction dir.

Constraint (23) requires that once RS $r$ associates with BS $b$ in direction dir, the paths from $b$ to $r$ must be selected by one or more than one MC.

Constraint (24) requires that there is exactly one path to be selected by an MC from the associated BS to RS $r$ only if the MC selects RS $r$ in direction dir.

There are two constructions in (24): first, every two RSs selected by an MC must associate with the same BS; second, the numbers of links of every two paths selected by an MC must be the same.

For each MC, every receiving RS $v$ on a link $u v$ in DL is installed in (26) and every transmitting RS $u$ on a link $u v$ in UL is installed in (27).

Constraint (28) requires that, if link $u v$ is on the path $p$ adopted by the MC $n$ to reach RS $r$ in direction dir, then $y_{n u v}^{\text {dir }}$ must be 1 .

(5) Link Capacity Constraints. The aggregate flow of link $u v$ in direction dir is restricted in (29).

(6) Integer Constraint. Constraints (30) are integer properties of the decision variables.

\section{Solution Approach and Computational Experiments}

4.1. Lagrangian Relaxation Techniques. By applying the Lagrangian Relaxation (LR) Method and the Subgradient Method to solve the complex problem, based on the problem formulation mentioned previously, the first step would be that the constraints of the primal problem are relaxed by using the LR Method [26]. In this step, we can not only determine a theoretical lower bound of the primal problem, but, can also glean some hints of feasible solutions captured by the primal problems. After iterations, the end result of the Lagrangian Relaxation Problem is guaranteed to a feasible solution by a feasible step which is satisfied with all constraints of the primal problem, if not, we have to make some modifications.

4.2. Getting Primal Feasible Heuristics. To obtain the primal feasible solutions for (IP 1), the first step is considered the solutions to the Lagrangian Relaxation. Two major decision variables, $\kappa_{n s}^{\mathrm{dir}}$ and $y_{n u v}^{\mathrm{dir}}$ are taken into consideration. According to $\kappa_{n s}^{\mathrm{dir}}$, the RS(s) (or BS) can be obtained to serve
MS $n$ selected in dir direction, and $y_{n u v}^{\mathrm{dir}}$ represents the link $u v$ which $n$ selected on the routing multicast tree in dir direction. In addition to $\kappa_{n s}^{\mathrm{dir}}$ and $y_{n u v}^{\mathrm{dir}}$, for the complexity of this problem including five $0-1$ integer decision variables, we still need other clues to help solving this problem in good quality. Thus, the coefficient $\mu_{n s 1}^{4}+\sum_{b \in B} \mu_{n s b 1}^{15}+P_{\min }^{N} \mu_{n s}^{5}+$ $\bar{M} \sum_{i \in R} \sum_{b \in B} \mu_{n i s b 1}^{16}-\pi_{s n}^{1}\left(\mu_{n s}^{5}+\mu_{n}^{7}-\mu_{n s}^{8}\right)$ of $\kappa_{n s}^{1}$ in DL, namely, $C_{\kappa}^{1} ; \mu_{n r 2}^{4}+\sum_{b \in B} \mu_{n r b 2}^{15}+P_{\min }^{R} \mu_{n r}^{6}+\bar{M} \sum_{i \in R} \sum_{b \in B} \mu_{n i r b 2}^{16}-\pi_{n r}^{2}\left(\mu_{n r}^{6}-\right.$ $\left.\mu_{n r}^{9}\right)+\mu_{n}^{14} \mathrm{BEP}\left(\pi_{n r}^{2}\right)$ of $\kappa_{n s}^{2}$ in UL, namely, $C_{\kappa}^{2}$ is introduced in our solution to sort $\kappa_{n s}^{\text {dir }}$ for further calculations.

The main purpose of determining the primal feasible heuristic is, in both DL and UL directions, and for each MS sorted by the distance to BS, to fully utilize the RSs built already to meet the BER requirement, and if not, to at least minimize the number of RSs necessary to reach the previous goal. The detailed procedure that decomposites the Lagrangian Relaxation Problem into several subproblems is described in the appendix.

4.3. Experiments Environment. In this session, we conduct several computational experiments to justify the proposed algorithms. Due to limitation of available experiment scenarios and parameters, we focus on IEEE 802.16j instead of LTE-A; it is easier to build the network based on realistic and operable environment parameters. In order to effectively analyze the physical operations of an 802.16j network, Table 3 lists all system parameters utilized in this research with reference to "Mobile WiMAX" published by WiMAX forum. Adaptive Modulation and Coding (AMS) applied in 802.16j is illustrated specifically in Table 4 with the same reference to "Mobile WiMAX."

In the meantime, and for the purpose of evaluating our solution of quality, two simple algorithms, minimum BER algorithm (MBA) and density-based algorithm (DBA), are implemented for comparison. The purpose of each MBA is, for each MS $n$, always to find the best paths that can generate the smallest BER value $n$ receives in DL and BS $b$ receives in UL. This algorithm will provide every transmission the minimum BER. The other one is DBA, the main concept would be the building of an RS with the first priority of the highest density area which is not served at the edges of coverage.

Path Loss Function [27]

$$
\begin{aligned}
\overline{P L(d)}(\mathrm{dB})= & 32.45+10 \times n \log f_{c}(\mathrm{MHz}) \\
& +10 \times n \log d(\mathrm{~km}),
\end{aligned}
$$

where $n$ is attenuation factor, $f_{c}$ is operation frequency, $d$ is distance.

Thermal Noise Function

$$
\begin{gathered}
N=K T_{0} B F, \quad \text { transfer into }(\mathrm{dB}): \\
N=-174(\mathrm{dBm})+10 \log _{10} B+F(\mathrm{~dB}),
\end{gathered}
$$

where $B$ is channel bandwidth, $F$ is noise figure. 
TABLE 3: System parameters [24, 25].

\begin{tabular}{lc}
\hline Parameters & Value \\
\hline Operation frequency & $2500 \mathrm{MHz}$ \\
Channel bandwidth & $10 \mathrm{MHz}$ \\
BS antenna gain & $15 \mathrm{dBi}$ \\
RS basic antenna gain & $5 \mathrm{dBi}$ \\
MS antenna gain & $-1 \mathrm{dBi}$ \\
BS noise figure & $4 \mathrm{~dB}$ \\
RS noise figure & $5 \mathrm{~dB}$ \\
MS noise figure & $7 \mathrm{~dB}$ \\
BS transmit power & $43 \mathrm{dBm}$ \\
RS basic transmit power & $33 \mathrm{dBm}$ \\
MS transmit power & $23 \mathrm{dBm}$ \\
RS config, set & 3 \\
Attenuation factor & 3.2 \\
Thermal noise figure & $-174 \mathrm{~dB}$ \\
Min. RS to RS SNR & $7.9515 \mathrm{~dB}$ \\
Min. SNR received by MS & $2.6505 \mathrm{~dB}$ \\
BER threshold & 0.0001 \\
Max. spatial diversity & 3 \\
Traffic required by MS (DL) & $1 \mathrm{Mbps}$ \\
Traffic required by MS (UL) & $0.5 \mathrm{Mbps}$ \\
BS capacity & $100 \mathrm{Mbps}$ \\
RS basic capacity & $15 \mathrm{Mbps}$ \\
RS fix cost & $1 \mathrm{M} \mathrm{dollars}$ \\
RS config. cost & $0.2 \mathrm{M} \mathrm{dollars}$ \\
\hline
\end{tabular}

In this research, the SNR function we apply is listed as follows:

$$
\operatorname{SNR}(\mathrm{dBm})=P_{t}+G_{t}+G_{r}-\overline{P L(d)}-N
$$

where $P_{t}$ is transmit power, $G_{t}$ is transmit gain, $G_{r}$ is receive gain, $\overline{P L(d)}$ is path loss function, $N$ is thermal noise function.

The BER evaluation functions we apply have been moderated with various modulation schemes $[28,29]$ are demonstrated the theoretical and simulated results of BER value in four different modulation schemes.

4.4. Experiment Scenarios. For the unique characteristics of this network deployment problem, the given circumstances are BS and MS locations, but RSs would be candidate locations. There is no RS built at the beginning. The word "topology" introduced in the following refers to the geographic distribution (the position) of locations where an RS could be built. Two types of topologies, grid and random, are proposed with different numbers of RS and MS in one BS environment to analyze the impact on deployment cost. We then apply different numbers of RS and MS with two BSs in a random topology to analyze the deployment in multiple BSs environment. Table 5 lists the experiment scenarios; Figures 5 and 6 show the graphic examples of grid and random networks. For each scenario, all MSs are guaranteed to have transmission paths and each scenario can be solved in our experiments. In these scenarios, BSs are at the center

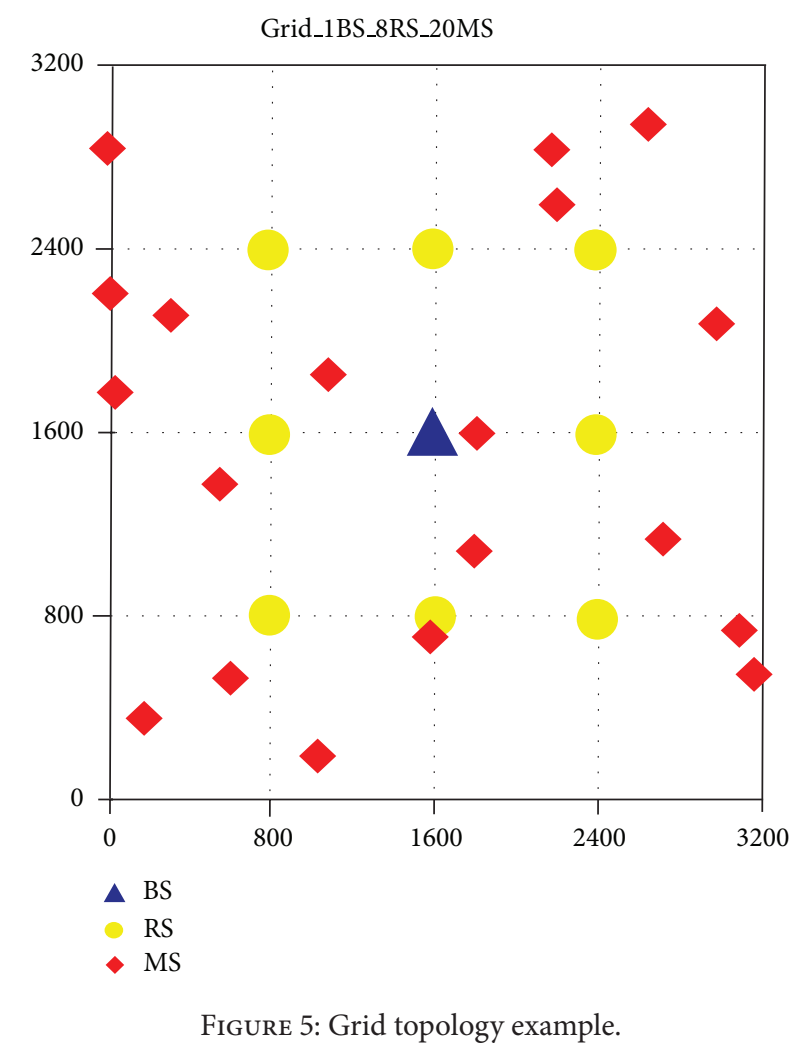

of the network, RSs are in Grid/Random topology, and MSs are in random topology.

4.5. Experiment Results. In Lagrangian relaxation approach, an upper bound (UB) of the problem, is the best primal feasible solution, while the solution to the Lagrangian dual problem guarantees the lower bound (LB) of the problem. By solving the Lagrangian dual problem iteratively and getting a primal feasible solution, we derive the LB and the UB, respectively. Thus, the gap between the UB and $\mathrm{LB}$, computed by $(\mathrm{UB}-\mathrm{LB}) / \mathrm{LB} \times 100 \%$, illustrates the quality (optimality) of the problem solution.

Figure 7 and Table 6 show the total deployment cost calculated by different algorithms within $1 \mathrm{BS}$ and grid RS topology configuration with different numbers of RS and MS are deployed, respectively. It is obvious that LR-based algorithm results in superior solution in comparison with MBA and DBA, especially when the RS number is large. Additionally, DBA has lower costs than MBA. This illustrates that the LR algorithm has a trend of choosing RS with large MS density instead of RS, which results in minimum BER.

In RS grid topology, for a given network scale, the distance of RS is the farthest locations from BS to receive signals under BER threshold should be included mandatorily. This phenomenon can be observed in Figures 8 and 9. The RS locations in grid topologies of $\mathrm{RS}=24$ exclude the RS locations in the same topologies of RS $=8$ where the about farthest locations BS can reach an RS. The costs in the scenarios of $\mathrm{RS}=24$ are all higher than in the scenarios of $\mathrm{RS}=8$ except MBA. We infer that this is because some MSs 
TABLE 4: Modulation and code rate [24, 25].

\begin{tabular}{lcccc}
\hline Modulation & Code rate & SNR & DL rate (Mbps) & UL rate $($ Mbps $)$ \\
\hline QPSK & $1 / 2$ CTC & SNR $\leq 9.4$ & 6.34 & 4.70 \\
QPSK & $3 / 4$ CTC & $9.4<$ SNR $\leq 11.2$ & 9.50 & 7.06 \\
16 QAM & $1 / 2$ CTC & $11.2<$ SNR $\leq 16.4$ & 12.67 & 9.41 \\
16 QAM & $3 / 4$ CTC & $16.4<$ SNR $\leq 18.2$ & 19.01 & 14.11 \\
64 QAM & 2/3 CTC & $18.2<$ SNR $\leq 22.7$ & 25.34 & 18.82 \\
64 QAM & 3/4 CTC & $22.7<$ SNR & 28.51 & 21.17 \\
\hline
\end{tabular}

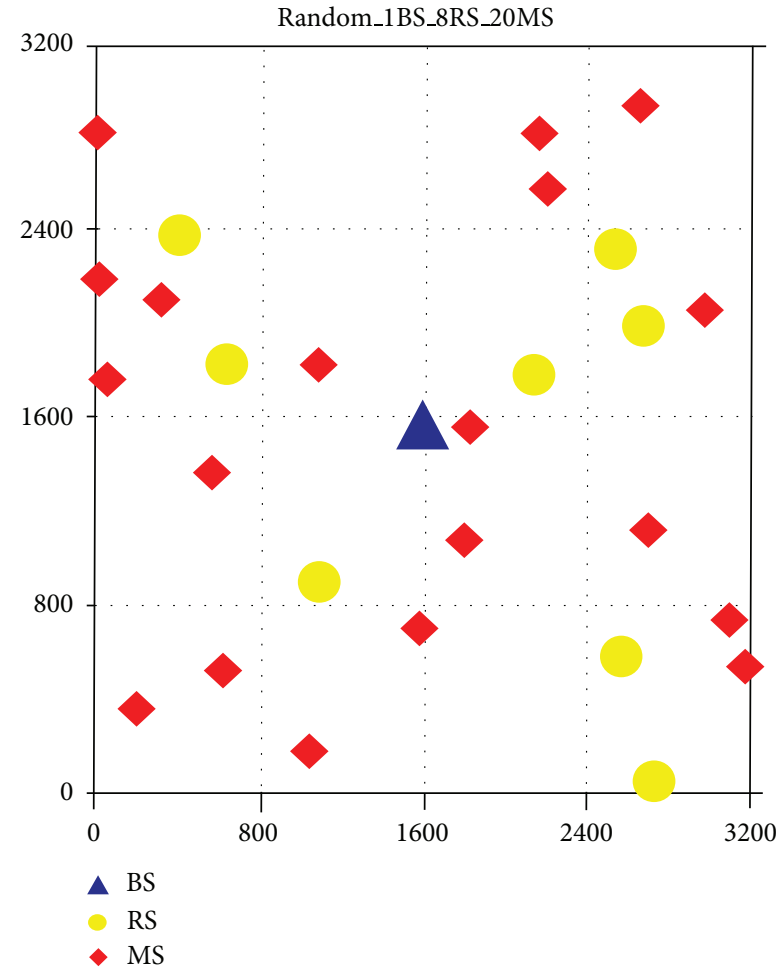

FIgURE 6: Random topology example.

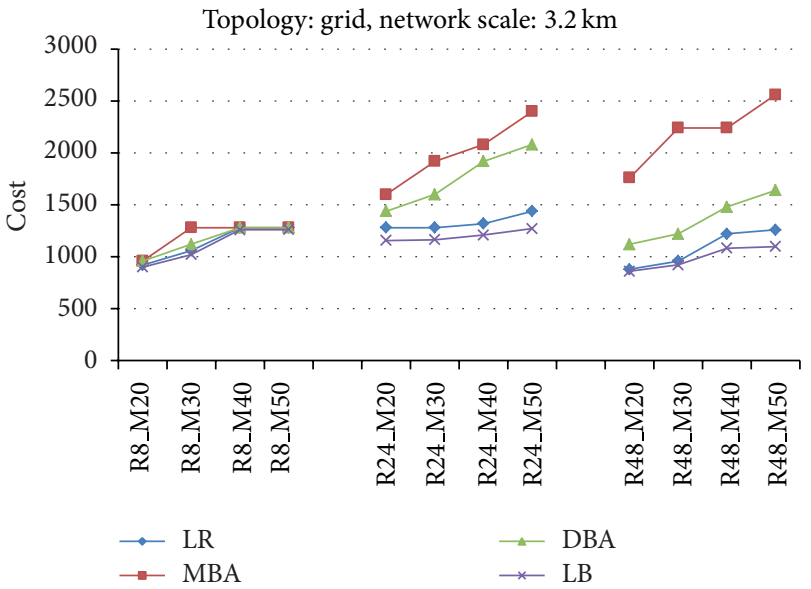

FIGURE 7: Deployment cost with different number of RS and MS (1 BS, grid, $3.2 \mathrm{~km}$ ).
TABLE 5: Experiment scenarios.

\begin{tabular}{lcccc}
\hline Topology & $\begin{array}{c}\text { Network } \\
\text { scale }\end{array}$ & No. of BS & No. of RS & No. of MS \\
\hline Grid & $3.2 \mathrm{~km}$ & 1 & $8,24,48$ & $20,30,40,50$ \\
Grid & $6.4 \mathrm{~km}$ & 1 & 24,48 & $20,30,40,50$ \\
Grid & $9.6 \mathrm{~km}$ & 1 & 80 & 20 \\
Random & $3.2 \mathrm{~km}$ & 1 & $8,24,48$ & $20,30,40,50$ \\
Random & $6.4 \mathrm{~km}$ & 2 & 16,48 & $40,60,80$ \\
\hline
\end{tabular}

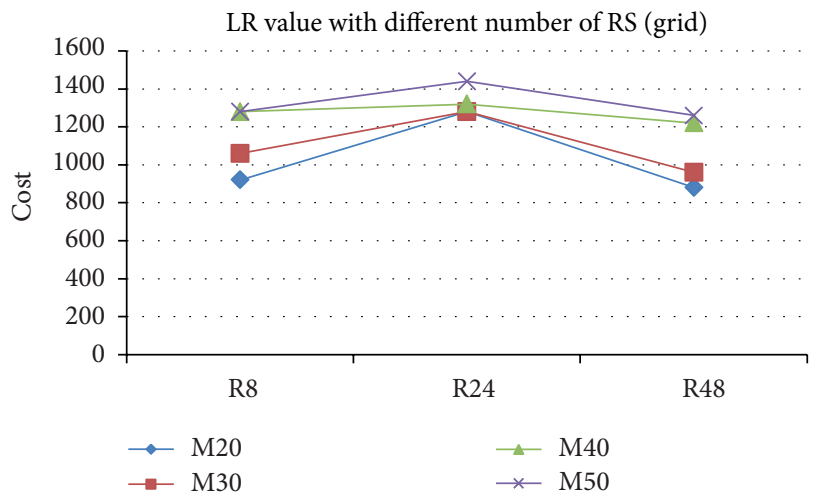

Figure 8: Deployment cost with different number of RS (1 BS, grid, $3.2 \mathrm{~km})$.

in $\mathrm{RS}=24$ need more hops than $\mathrm{RS}=8$ to reach the $\mathrm{BS}$, thus inducing costs.

From Figures 10 and 11, we can come to the conclusion that with a fixed number of MSs, total deployment costs are reduced with an increasing number of RSs. Meanwhile, with a fixed number of RSs, total deployment costs are reduced with an increase in the number of MSs.

Figure 10 and Table 7 show total deployment costs as calculated by different algorithms through $1 \mathrm{BS}$ with different numbers of RS and MS in a random topology. Again, it is obvious that the Lagrangian Relaxation-based algorithm receives better solution of quality in comparison with $\mathrm{MBA}$ and DBA (bold font), particularly so with a large number of RSs.

Figures 11 and 12 indicate the same conclusion in grid topology. With a fixed number of MSs, total deployment costs are reduced with an increase in the number of RSs. At the same time, with a fixed number of RSs, total deployment costs are reduced with an increase in the number of MSs. 
TABLE 6: Algorithm comparison (1 BS, grid, $3.2 \mathrm{~km}$ ).

\begin{tabular}{|c|c|c|c|c|c|c|c|c|}
\hline No. of RS & No. of $\mathrm{MC}$ & LB & UB & GAP (\%) & MBA & Imp. ratio of MBA (\%) & DBA & Imp. ratio of DBA (\%) \\
\hline 8 & 20 & 901.7678 & 920 & 1.98176 & 960 & 4.347826 & 960 & 4.347826 \\
\hline 8 & 30 & 1020.242 & 1060 & 3.750792 & 1280 & 20.75472 & 1120 & 5.660377 \\
\hline 8 & 40 & 1258.947 & 1280 & 1.644797 & 1280 & $\mathbf{0}$ & 1280 & $\mathbf{0}$ \\
\hline 8 & 50 & 1260.484 & 1280 & 1.524727 & 1280 & $\mathbf{0}$ & 1280 & $\mathbf{0}$ \\
\hline 24 & 20 & 1156.286 & 1280 & 9.665148 & 1600 & 25 & 1440 & 12.5 \\
\hline 24 & 30 & 1164.774 & 1280 & 9.002031 & 1920 & 50 & 1600 & 25 \\
\hline 24 & 40 & 1208.743 & 1320 & 8.428545 & 2080 & 57.57576 & 1920 & 45.45455 \\
\hline 24 & 50 & 1269.846 & 1440 & 11.81623 & 2400 & 66.66667 & 2080 & 44.44444 \\
\hline 48 & 20 & 860.6118 & 880 & 2.203205 & 1760 & 100 & 1120 & 27.27273 \\
\hline 48 & 30 & 921.4716 & 960 & 4.013375 & 2240 & 133.3333 & 1220 & 27.08333 \\
\hline 48 & 40 & 1082.548 & 1220 & 11.2666 & 2240 & 83.60656 & 1480 & 21.31148 \\
\hline 48 & 50 & 1098.812 & 1260 & 12.79272 & 2560 & 103.1746 & 1640 & 30.15873 \\
\hline
\end{tabular}

TABLE 7: Algorithm comparison (1 BS, random, $3.2 \mathrm{~km}$ ).

\begin{tabular}{lcccccccc}
\hline No. of RS & No. of MC & LB & UB & GAP (\%) & MBA & Imp. ratio of MBA (\%) & DBA & Imp. ratio of DBA (\%) \\
\hline 8 & 20 & 867.3459 & 900 & 3.628233 & 960 & $\mathbf{6 . 6 6 6 6 6 7}$ & 960 & $\mathbf{6 . 6 6 6 6 6 7}$ \\
8 & 30 & 850.3321 & 900 & 5.518652 & 960 & $\mathbf{6 . 6 6 6 6 6 7}$ & 960 & $\mathbf{6 . 6 6 6 6 6 7}$ \\
8 & 40 & 846.2536 & 900 & 5.971822 & 1120 & $\mathbf{2 4 . 4 4 4 4 4}$ & 960 & $\mathbf{6 . 6 6 6 6 6 7}$ \\
8 & 50 & 909.7847 & 980 & 7.164823 & 1280 & $\mathbf{3 0 . 6 1 2 2 4}$ & 1020 & $\mathbf{4 . 0 8 1 6 3 3}$ \\
24 & 20 & 811.1707 & 860 & 5.67783 & 1600 & $\mathbf{8 6 . 0 4 6 5 1}$ & 960 & $\mathbf{1 1 . 6 2 7 9 1}$ \\
24 & 30 & 798.1251 & 860 & 7.19476 & 1920 & $\mathbf{1 2 3 . 2 5 5 8}$ & 1020 & $\mathbf{1 8 . 6 0 4 6 5}$ \\
24 & 40 & 805.3412 & 900 & 10.51765 & 2080 & $\mathbf{1 3 1 . 1 1 1 1}$ & 1020 & $\mathbf{1 3 . 3 3 3 3 3}$ \\
24 & 50 & 860.9539 & 980 & 12.14756 & 2400 & $\mathbf{1 4 4 . 8 9 8}$ & 1340 & $\mathbf{3 6 . 7 3 4 6 9}$ \\
48 & 20 & 734.8455 & 820 & 10.3847 & 1760 & $\mathbf{1 1 4 . 6 3 4 1}$ & 1020 & $\mathbf{2 4 . 3 9 0 2 4}$ \\
48 & 30 & 766.4685 & 860 & 10.87563 & 1820 & $\mathbf{1 1 1 . 6 2 7 9}$ & 1080 & $\mathbf{2 5 . 5 8 1 4 2}$ \\
48 & 40 & 744.6947 & 880 & 15.3756 & 2260 & $\mathbf{1 5 6 . 8 1 8 2}$ & 1140 & $\mathbf{2 9 . 5 4 5 4 5}$ \\
48 & 50 & 768.6480 & 920 & 16.4513 & 2420 & $\mathbf{1 6 3 . 0 4 3 5}$ & 1260 & $\mathbf{3 6 . 9 5 6 5 2}$ \\
\hline
\end{tabular}

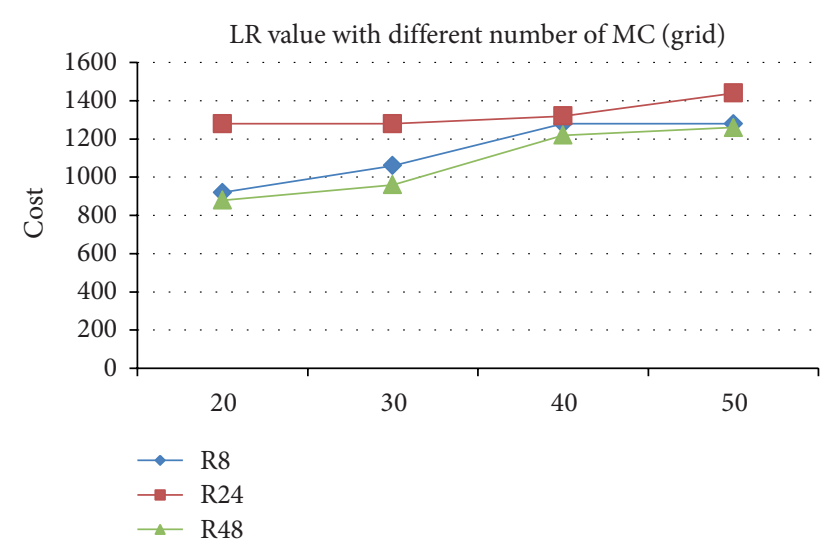

FIGURE 9: Deployment cost with different number of MS (1 BS, grid, $3.2 \mathrm{~km})$.

In random topology, it is difficult to generate a network capable of satisfying every MS's transmission when a few RSs (ex. $\mathrm{RS}=8$ ) are deployed. In general, RSs are not distributed uniformly enough to fully cover all MSs. Figure 13 and Table 8 show total deployment costs calculated by different algorithms under 2 BS and random topology, with different

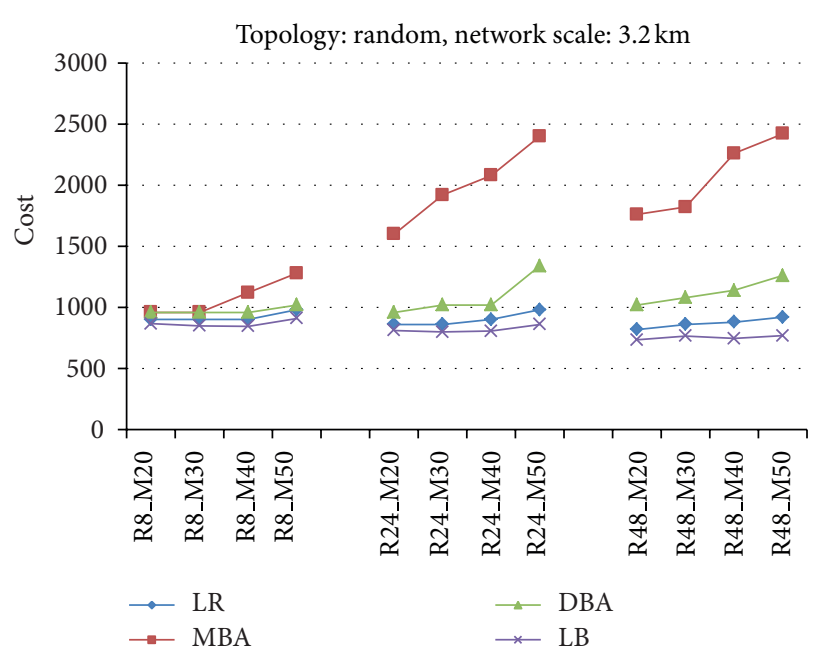

FIGURE 10: Deployment cost with different number of RS and MS (1 BS, random, $3.2 \mathrm{~km}$ ).

number of RS and MS. We come to the same conclusion: the Lagrangian Relaxation-based algorithm still gets better 
TABle 8: Algorithm comparison (2 BSs, random, $6.4 \mathrm{~km}$ ).

\begin{tabular}{lcccccccc}
\hline No. of RS & No. of MC & LB & UB & GAP (\%) & MBA & Imp. ratio of MBA (\%) & DBA & Imp. ratio of DBA (\%) \\
\hline 16 & 40 & 1542.505 & 1620 & 4.78362 & 1760 & $\mathbf{8 . 6 4 1 9 7 5}$ & 1680 & $\mathbf{3 . 7 0 3 7 0 4}$ \\
16 & 60 & 1726.22 & 1840 & 6.18371 & 2240 & $\mathbf{2 1 . 7 3 9 1 3}$ & 1840 & $\mathbf{0}$ \\
16 & 80 & 1737.373 & 1920 & 9.5118 & 2400 & $\mathbf{2 5}$ & 2020 & $\mathbf{5 . 2 0 8 3 3 3}$ \\
48 & 40 & 1409.38 & 1540 & 8.48179 & 3040 & $\mathbf{9 7 . 4 0 2 6}$ & 1760 & $\mathbf{1 4 . 2 8 5 7 1}$ \\
48 & 60 & 1533.7687 & 1720 & 10.8274 & 3360 & $\mathbf{9 5 . 3 4 8 8 4}$ & 1940 & $\mathbf{1 2 . 7 9 0 7}$ \\
48 & 80 & 1550.1775 & 1820 & 14.82541 & 3840 & $\mathbf{1 1 0 . 9 8 9}$ & 2280 & $\mathbf{2 5 . 2 7 4 7 3}$ \\
\hline
\end{tabular}

TABLE 9: Experiment results (1 BS, grid, $6.4 \mathrm{~km}$ ).

\begin{tabular}{lcccccccc}
\hline No. of RS & No. of MC & LB & UB & GAP (\%) & MBA & Imp. ratio of MBA (\%) & DBA & Imp. ratio of DBA (\%) \\
\hline 8 & 20 & N/A & N/A & N/A & N/A & N/A & N/A & N/A \\
8 & 30 & N/A & N/A & N/A & N/A & N/A & N/A & N/A \\
8 & 40 & N/A & N/A & N/A & N/A & N/A & N/A & N/A \\
8 & 50 & N/A & N/A & N/A & N/A & $\mathbf{1 6 . 2 3 9 3 2}$ & 2420 & $\mathbf{3 . 4 1 8 8 0 3}$ \\
24 & 20 & 2155.106 & 2340 & 7.901457 & 2720 & $\mathbf{4 1 . 9 3 5 4 8}$ & 2840 & $\mathbf{1 4 . 5 1 6 1 3}$ \\
24 & 30 & 2212.838 & 2480 & 10.77267 & 3520 & $\mathbf{7 0 . 2 1 2 7 7}$ & 3360 & $\mathbf{1 9 . 1 4 8 9 4}$ \\
24 & 40 & 2469.994 & 2820 & 12.41156 & 4800 & $\mathbf{5 8 . 1 3 9 5 3}$ & 4480 & $\mathbf{3 0 . 2 3 2 5 6}$ \\
24 & 50 & 2699.765 & 3440 & 21.51847 & 5440 & $\mathbf{6 9 . 4 1 1 7 6}$ & 1960 & $\mathbf{1 5 . 2 9 4 1 2}$ \\
48 & 20 & 1537.11 & 1700 & 9.581763 & 2880 & $\mathbf{9 3 . 1 0 3 4 5}$ & 2640 & $\mathbf{1 3 . 7 9 3 1}$ \\
48 & 30 & 2059.57 & 2320 & 11.22543 & 4480 & $\mathbf{1 0 5 . 8 8 2 4}$ & 3480 & $\mathbf{2 7 . 9 4 1 1 8}$ \\
48 & 40 & 2309.617 & 2720 & 15.08761 & 5600 & $\mathbf{9 0 . 2 4 3 9}$ & 4880 & $\mathbf{4 8 . 7 8 0 4 9}$ \\
48 & 50 & 2445.661 & 3280 & 25.43715 & 6240 & &
\end{tabular}

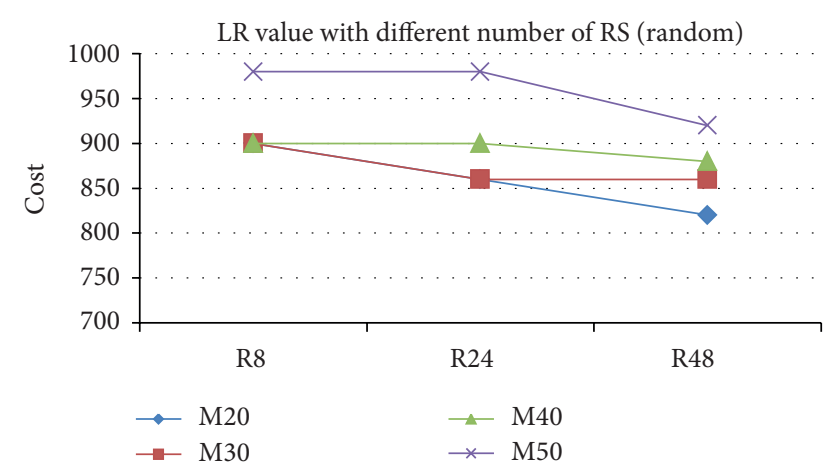

FIGURE 11: Deployment cost with different number of RS (1BS, random, $3.2 \mathrm{~km}$ ).

solution of quality in comparison with MBA and DBA, more so with a large number of RSs.

If these scenarios experimented previously are double the size of those in which $\mathrm{BS}=1$, how the result is. With random topology in $\mathrm{BS}=1$, it is also difficult to get a feasible network when RS number is small (ex. RS $=16$ here). Figure 13 illustrates total deployment costs in random topologies with $\mathrm{BS}=1$ and $\mathrm{BS}=2$. Since the RS locations are different in both conditions, it would be fruitless to compare their costs. However, it is still obvious that the gaps are all larger in every scenarios in $\mathrm{BS}=2$ than in $\mathrm{BS}=1$ for network complexity.

Figure 14 and Table 9 show the scenario of 1 BS, with different number of RS and MS in a grid topology with a $6.4 \mathrm{~km}$ network scale. Since the network $(6.4 \mathrm{~km})$ is larger

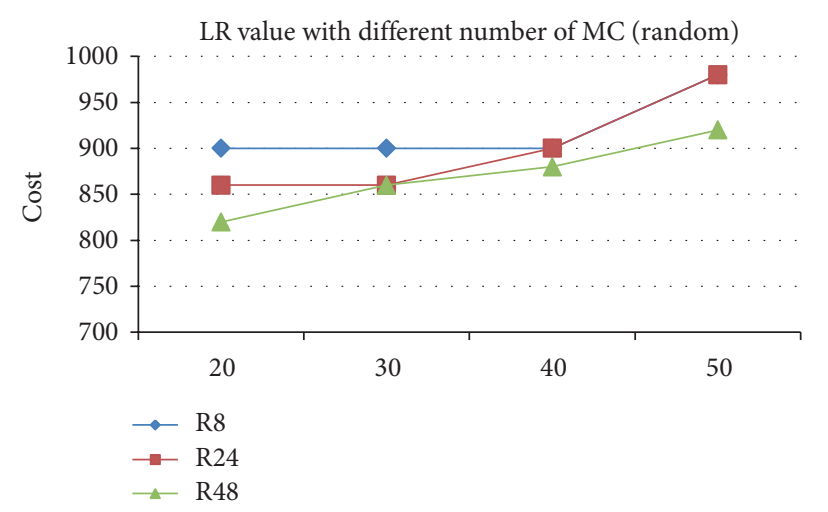

FIGURE 12: Deployment cost with different number of MS (1 BS, random, $3.2 \mathrm{~km}$ ).

than that of previous experiments $(3.2 \mathrm{~km}), 8 \mathrm{RSs}$ is no longer sufficient to fulfill all transmissions. Therefore, 24 RSs (two layers from the BS) becomes the smallest size of this network scale.

Figure 15 demonstrates the deployment costs of $20 \mathrm{MSs}$ with various numbers of RS among three kinds of network scale, $3.2 \mathrm{~km}$ with 8 RSs (1 layer from the BS), $6.4 \mathrm{~km}$ with $24 \mathrm{RSs}$, and $9.6 \mathrm{~km}$ with $80 \mathrm{RSs}$ (4 layers from the BS). As explained previously, in the $6.4 \mathrm{~km}$ network scale the smallest grid size is $24 \mathrm{RSs}$ ( 2 layers from the BS). One can see the same situation in $9.6 \mathrm{~km}$, with the smallest grid size being 80 RSs ( 3 layers from the BS). 


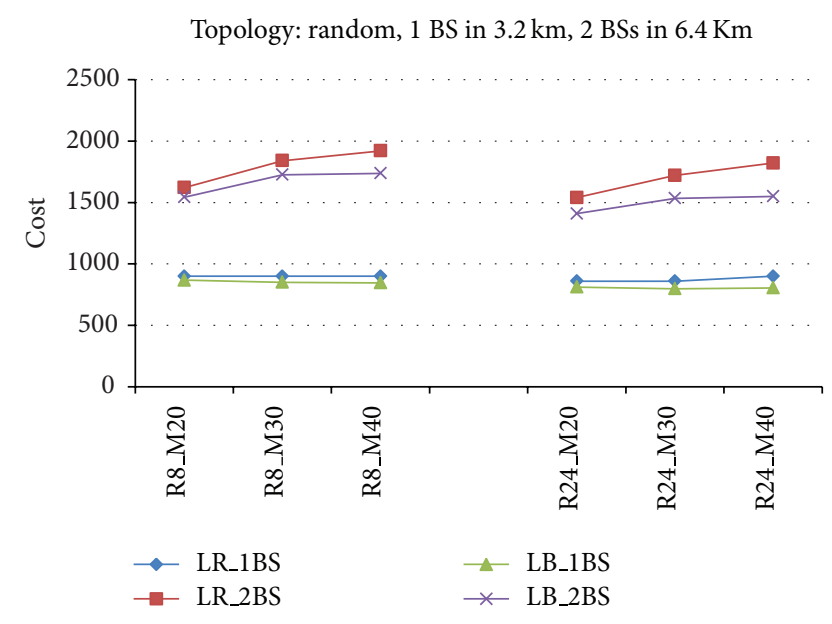

FIGURE 13: Deployment cost with different number of BS in random topology.

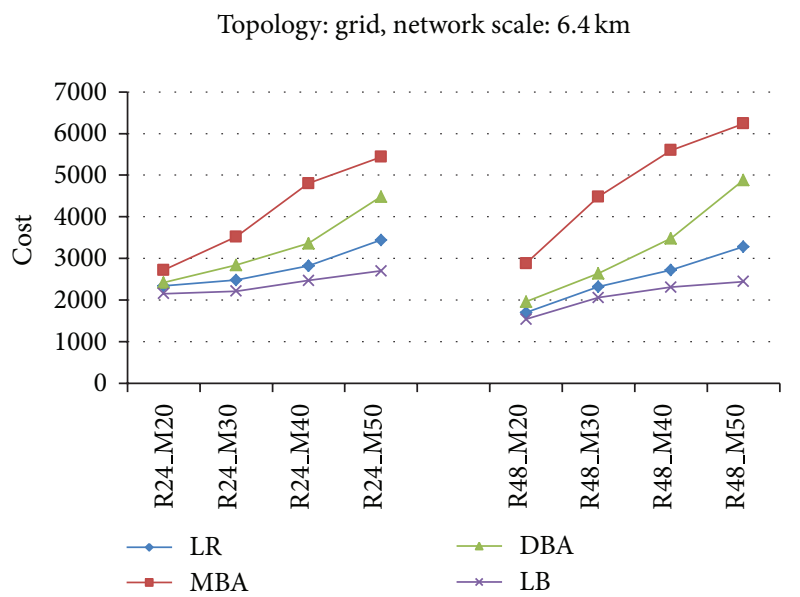

FIGURE 14: Deployment cost with different number of RS and MS (1 BS, gird, $6.4 \mathrm{~km}$ ).

\section{Conclusions and Future Work}

With 3G technology established, it was obvious that the traffic is increased significantly, but the average revenue per user (ARPU) is decreased very fast. The business challenges of operators would be that increasing revenues by finding other solutions more efficiency and effectiveness. But the network development of a new $4 \mathrm{G}$ system started to be investigated and made huge investments of macro base station deployments. In one early investigation which took relays would be able to speed up extend services and expanded market share at this stage economically. So, the operators can make new revenues and cost reduction balance.

Although our experiments do not cover large network scales with large number of RSs and MSs for the restrictions of computational capabilities, these model approaches can nevertheless be regarded as useful engineering guidelines for future LTE-A relay network development.

In this paper, we stand for an operator to propose a solution that determines where and how many relays should

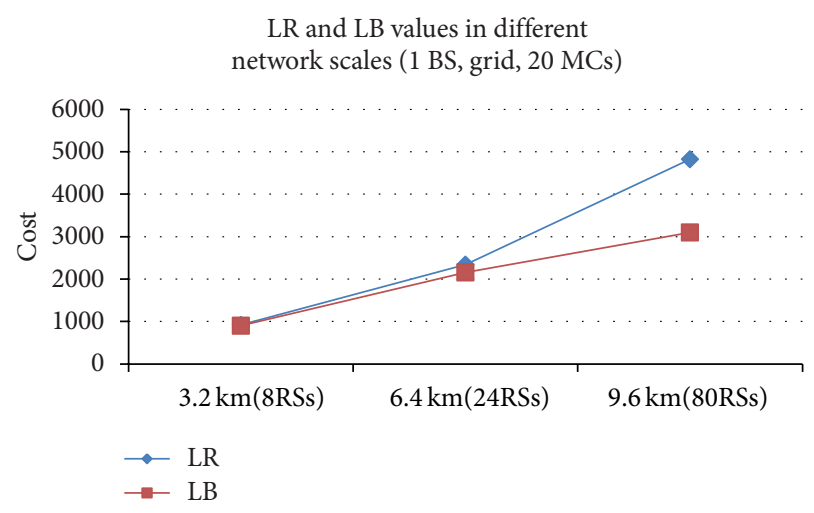

Figure 15: Deployment cost and lower bound (LB) values in different network scales (1 BS, grid, $20 \mathrm{MSs}$ ).

be deployed in the planning stages to minimize the development cost. In the planning stages, we not only derive a Multicast Tree routing algorithm to both determine and fulfill the QoS requirements and also enhance throughput on both down-link and up-link communications, but we also utilize the Lagrangian Relaxation Method in conjunction with optimization-based heuristics and conduct computational experiments to evaluate the performance of the proposed algorithms.

Our contributions in this research can be divided into three parts. First, we have constructed the network architecture with multicast tree routing concepts. Secondly, we proposed a precise mathematical expression to model the network architecture problem. This is not an intuitive mathematical model for considering the solvability of this problem. We have designed the entire model not only to be solvable but also to not violate the physical meanings. Finally, we provide the lagrangian relaxation and optimization-Based algorithms to solve this problem; we prove it to have superior quality after verification with other simple algorithms and lower bound value. This optimal solution is a good strategic method to minimize the CAPEX of operators to build up a relay network with more efficiency and effectiveness and the QoS can be guaranteed.

\section{Appendix}

Solution Approach. The wireless relay deployment problem is emulated as a mixed integer and linear programming (MILP) problem. To solve this problem, the optimal development cost for network planning is minimized to relay selection constraints, nodal, and link capacity constraints, cooperatively relaying constraints, and routing constraints for both UL and DL transmissions. The Lagrangian Relaxation method is proposed in conjunction with the optimization-based heuristics to solve the problem. The primal problem (IP 1) is transformed into the following Lagrangian Relaxation Problem, where constraints (3), (4), (5), (6), (10), (11), (12), (14), (15), (16), (17), (19), (20), (21), (22), (23), (25), (26), (27), (28), and (29) are relaxed by introducing Lagrangian multiplier vector $\mu_{1} \sim \mu_{22}$. 


\section{Optimal Problem. One has}

$$
\begin{aligned}
& Z_{D}\left(\mu_{1}, \mu_{2}, \mu_{3}, \mu_{4}, \mu_{5}, \mu_{6}, \mu_{7}, \mu_{8}, \mu_{9}, \mu_{10}, \mu_{11}, \mu_{12}, \mu_{13}, \mu_{14}, \mu_{15}, \mu_{16}, \mu_{17}, \mu_{18}, \mu_{19}, \mu_{20}, \mu_{21}, \mu_{22}\right) \\
& =\min \sum_{r \in R} \sum_{k \in K}\left(\psi_{r}+\Phi_{r}(k)\right) \eta_{r k}+\sum_{r \in R} \sum_{\text {dir } \in \text { DIR }} \mu_{r \text { dir }}^{1}\left[\sum_{b \in B} h_{r b}^{\mathrm{dir}}-\sum_{k \in K} \eta_{r k}\right] \\
& +\sum_{r \in R} \mu_{r}^{2}\left[\sum_{u \in\{R \cup B\}} \sum_{n \in N} y_{n u r}^{1} \theta_{n}^{1}+\sum_{w \cup\{R \cup B\}} \sum_{n \in N} y_{n r w}^{2} \theta_{n}^{2}-\sum_{k \in K} \eta_{r k} \overline{C_{r}(k)}\right] \\
& +\sum_{b \in B} \mu_{b}^{3}\left[\sum_{r \in R} \sum_{n \in N} y_{n b r}^{1} \theta_{n}^{1}+\sum_{i \in R} \sum_{n \in N} y_{n i b}^{2} \theta_{n}^{2}+\sum_{n \in N} \sum_{\operatorname{dir} \in \mathrm{DIR}} \kappa_{n b}^{\mathrm{dir}} \theta_{n}^{\mathrm{dir}}-\overline{C_{b}}\right] \\
& +\sum_{n \in N} \sum_{r \in R} \sum_{\operatorname{dir} \in \operatorname{DIR}} \mu_{n r \operatorname{dir}}^{4}\left[\kappa_{n r}^{\mathrm{dir}}-\sum_{k \in K} \eta_{r k}\right]+\sum_{n \in N} \sum_{s \in\{R \cup B\}} \mu_{n s}^{5}\left[\kappa_{n s}^{1} P_{\min }^{N}-\kappa_{n s}^{1} \pi_{s n}^{1}\right] \\
& +\sum_{n \in N} \sum_{s \in\{R \cup B\}} \mu_{n s}^{6}\left[\kappa_{n s}^{2} P_{\min }^{R}-\kappa_{n s}^{2} \pi_{n s}^{2}\right]+\sum_{n \in N} \mu_{n}^{7}\left[\omega_{n}-\sum_{s \in\{R \cup B\}} \kappa_{n s}^{1} \pi_{s n}^{1}\right] \\
& +\sum_{n \in N} \sum_{s \in\{R \cup B\}} \mu_{n s}^{8}\left[\kappa_{n s}^{1} \pi_{s n}^{1}-\sum_{k \in K} \eta_{s k}\left(P\left(\rho_{s}^{1}(k), D_{s n}, \tau\right)-P_{N}(n)\right)\right] \\
& +\sum_{n \in N} \sum_{s \in\{R \cup B\}} \mu_{n s}^{9}\left[\kappa_{n s}^{2} \pi_{n s}^{2}-\sum_{k \in K} \eta_{s k}\left(P\left(\rho_{n}^{N}, D_{n s}, \tau\right)-P_{N}(s)\right)\right] \\
& +\sum_{n \in N} \sum_{u \in\{R \cup B\}} \sum_{v \in\{R \cup B\}} \sum_{\operatorname{dir} \in \mathrm{DIR}} \mu_{n u v \operatorname{dir}}^{10}\left[y_{n u v}^{\mathrm{dir}} P_{\min }^{R}-y_{n u v}^{\mathrm{dir}} \phi_{u v}^{\mathrm{dir}}\right]+\sum_{n \in N} \sum_{v \in\{R \cup B\}} \mu_{n v}^{11}\left[\varepsilon_{n v}-\sum_{u \in\{R \cup B\}} y_{n u v}^{2} \phi_{u v}^{2}\right] \\
& +\sum_{n \in N} \sum_{u \in\{R \cup B\}} \sum_{v \in\{R \cup B\}} \sum_{\operatorname{dir} \in \operatorname{DIR}} \mu_{n u v \operatorname{dir}}^{12}\left[y_{n u v}^{\mathrm{dir}} \phi_{u v}^{\mathrm{dir}}-\sum_{k \in K} \eta_{u k}\left(P\left(\rho_{u}^{\mathrm{dir}}(k), D_{u v}, \tau\right)-P_{N}(v)\right)\right] \\
& +\sum_{n \in N} \mu_{n}^{13}\left[\sum_{u \in\{R \cup B\}} \sum_{v \in\{R \cup B\}} y_{n u v}^{1} \mathrm{BEP}_{v}\left(\phi_{u v}^{1}\right)+\mathrm{BEP}_{n}\left(\omega_{n}\right)-\mathrm{BEP}^{1}\right] \\
& +\sum_{n \in N} \mu_{n}^{14}\left[\sum_{v \in\{R \cup B\}} \operatorname{BEP}_{v}\left(\varepsilon_{n v}\right)+\sum_{s \in\{R \cup B\}} \operatorname{BEP}_{s}\left(\kappa_{n s}^{2} \pi_{n s}^{2}\right)-\mathrm{BEP}^{2}\right]+\sum_{n \in N} \sum_{r \in R} \sum_{b \in B} \sum_{\operatorname{dir} \in \mathrm{DIR}} \mu_{n r b d i r}^{15}\left[\kappa_{n r}^{\mathrm{dir}}-h_{r b}^{\mathrm{dir}}\right] \\
& +\sum_{n \in N} \sum_{i \in R} \sum_{j \in R} \sum_{b \in B} \sum_{\mathrm{dir} \in \mathrm{DIR}} \mu_{n i j b \operatorname{dir}}^{16}\left[\sum_{p \in P_{b i}} \sum_{u \in\{R \cup B\}} \sum_{v \in\{R \cup B\}} x_{n i p}^{\mathrm{dir}} \delta_{p u v}-\sum_{p \in P_{b j}} \sum_{u \in\{R \cup B\}} \sum_{v \in\{R \cup B\}} x_{n j p}^{\mathrm{dir}} \delta_{p u v}-\left(1-\kappa_{n j}^{\mathrm{dir}}\right) \bar{M}\right] \\
& +\sum_{n \in N} \sum_{u \in\{R \cup B\}} \sum_{v \in\{R \cup B\}} \mu_{n u v}^{17}\left[y_{n u v}^{1}-\sum_{k \in K} \eta_{v k}\right]+\sum_{n \in N} \sum_{u \in\{R \cup B\}} \sum_{v \in\{R \cup B\}} \mu_{n u v}^{18}\left[y_{n u v}^{2}-\sum_{k \in K} \eta_{u k}\right] \\
& +\sum_{n \in N} \sum_{r \in R} \sum_{u \in\{R \cup B\}} \sum_{v \in\{R \cup B\}} \sum_{\mathrm{dir} \in \mathrm{DIR}} \mu_{n r u v \operatorname{dir}}^{19}\left[\sum_{b \in B} \sum_{p \in P_{b r}} x_{n r p}^{\mathrm{dir}} \delta_{p u v}-y_{n u v}^{\mathrm{dir}}\right] \\
& +\sum_{u \in\{R \cup B\}} \sum_{v \in\{R \cup B\}} \sum_{\operatorname{dir} \in \mathrm{DIR}} \mu_{u v \mathrm{dir}}^{21}\left[\sum_{n \in N} y_{n u v}^{\mathrm{dir}} \theta_{n}^{\mathrm{dir}}-C_{u v}\left(\phi_{u v}^{\mathrm{dir}}\right)\right]+\sum_{n \in N} \sum_{r \in R} \sum_{b \in B} \sum_{\mathrm{dir} \in \mathrm{DIR}} \mu_{n r b \mathrm{dir}}^{22}\left[h_{r b}^{\mathrm{dir}}-\sum_{p \in P_{b r}} x_{n r p}^{\mathrm{dir}}\right],
\end{aligned}
$$


subject to

$$
\begin{aligned}
& \sum_{k \in K} \eta_{r k} \leq 1 \quad \forall r \in R, \\
& \sum_{b \in B} h_{r b}^{\mathrm{dir}} \leq 1 \quad \forall r \in R, \operatorname{dir} \in \mathrm{DIR}, \\
& \sum_{b \in B} \kappa_{n b}^{\mathrm{dir}}+\kappa_{n r}^{\mathrm{dir}} \leq 1 \quad \forall n \in N, \\
& r \in R, \quad \text { dir } \in \text { DIR, } \\
& 1 \leq \sum_{s \in\{R \cup B\}} \kappa_{n s}^{\mathrm{dir}} \forall n \in N \text {, } \\
& s \in\{R \cup B\}, \quad \operatorname{dir} \in \mathrm{DIR}, \\
& \sum_{r \in R} \kappa_{n r}^{\mathrm{dir}} \leq \mathrm{SD}^{\mathrm{dir}} \quad \forall n \in N, \operatorname{dir} \in \mathrm{DIR}, \\
& 0 \leq \pi_{s n}^{\mathrm{dir}} \leq \bar{\pi} \quad \forall s \in\{R \cup B\}, \\
& n \in N, \quad \operatorname{dir} \in \text { DIR, } \\
& 0 \leq \phi_{u v}^{\mathrm{dir}} \leq \bar{\phi} \quad \forall u, v \in\{R \cup B\}, \operatorname{dir} \in \mathrm{DIR} \text {, } \\
& \sum_{b \in B} \sum_{p \in P_{b r}} x_{n r p}^{\mathrm{dir}} \leq 1 \quad \forall n \in N \text {, } \\
& r \in R, \quad \operatorname{dir} \in \mathrm{DIR}, \\
& \eta_{r k}=0 \text { or } 1 \quad \forall r \in R, k \in K \text {, } \\
& h_{r b}^{\text {dir }}=0 \text { or } 1 \quad \forall r \in R, \\
& b \in B, \quad \text { dir } \in \text { DIR, } \\
& \kappa_{n s}^{\text {dir }}=0 \text { or } 1 \quad \forall n \in N \text {, } \\
& s \in\{R \cup B\}, \quad \operatorname{dir} \in \mathrm{DIR}, \\
& x_{n r p}^{\mathrm{dir}}=0 \text { or } 1 \quad \forall p \in P_{b r}, b \in B \text {, } \\
& r \in R, \quad n \in N, \\
& y_{n u v}^{\mathrm{dir}}=0 \text { or } 1 \quad \forall n \in N \text {, } \\
& u, v \in\{R \cup B\}, \quad \operatorname{dir} \in \text { DIR. }
\end{aligned}
$$

Subproblem 1 (related to decision variable $\eta_{r k}$ ). One has

$$
\begin{aligned}
& Z_{\text {sub3.1 }}\left(\mu_{1}, \mu_{2}, \mu_{4}, \mu_{8}, \mu_{9}, \mu_{12}, \mu_{17}, \mu_{18}\right) \\
& =\min \left\{\sum _ { r \in R } \sum _ { k \in K } \left[\psi_{r}+\Phi_{r}(k)-\sum_{\operatorname{dir} \in \operatorname{DIR}} \mu_{r \operatorname{dir}}^{1}-\mu_{r}^{2} \overline{C_{r}(k)}\right.\right. \\
& +\sum_{n \in N}\left[-\sum_{\operatorname{dir} \in \operatorname{DIR}} \mu_{n r \operatorname{dir}}^{4}\right. \\
& -\mu_{n r}^{8}\left(P\left(\rho_{r}^{1}(k), D_{r n}, \tau\right)-P_{N}(n)\right) \\
& -\mu_{n r}^{9}\left(P\left(\rho_{n}^{N}, D_{n r}, \tau\right)-P_{N}(r)\right) \\
& -\sum_{u \in\{R \cup B\}}\left(\mu_{n u r}^{17}+\mu_{n r u}^{18}\right) \\
& -\sum_{v \in\{R \cup B\}} \sum_{\operatorname{dir} \in \operatorname{DIR}} \mu_{n r v \operatorname{dir}}^{12}
\end{aligned}
$$

subject to

$$
\begin{gathered}
\sum_{k \in K} \eta_{r k} \leq 1 \quad \forall r \in R, \\
\eta_{r k}=0 \text { or } 1 \quad \forall r \in R, k \in K .
\end{gathered}
$$

Because the configuration of BS is constant, (Sub 3.1) can be further decomposed into $|R|$ independent subproblems. For each candidate location $r$ :

$$
\begin{aligned}
\min \left\{\sum _ { k \in K } \left[\psi_{r}+\Phi_{r}(k)\right.\right. & -\sum_{\text {dir } \in \text { DIR }} \mu_{r \text { dir }}^{1}-\mu_{r}^{2} \overline{C_{r}(k)} \\
+\sum_{n \in N}[ & -\sum_{\text {dir } \in \text { DIR }} \mu_{n r \mathrm{dir}}^{4} \\
& -\mu_{n r}^{8}\left(P\left(\rho_{r}^{1}(k), D_{r n}, \tau\right)-P_{N}(n)\right) \\
& -\mu_{n r}^{9}\left(P\left(\rho_{n}^{N}, D_{n r}, \tau\right)-P_{N}(r)\right) \\
& -\sum_{u \in\{R \cup B\}}\left(\mu_{n u r}^{17}+\mu_{n r u}^{18}\right) \\
& -\sum_{v \in\{R \cup B\} \text { dir } \in \text { DIR }} \mu_{n r v d i r}^{12} \\
\times\left(P\left(\rho_{r}^{\text {dir }}(k), D_{r v}, \tau\right)\right. & \left.\left.\left.\quad-P_{N}(v)\right)\right] \eta_{r k}\right\} \\
\text { subject to (A.3a) and (A.3b). } & \quad \text { Sub 3.1.1 }
\end{aligned}
$$


For each (Sub 3.1.1), find the configuration $k$ corresponding to the smallest coefficient value of $\eta_{r k}$. If the coefficient is negative, then set $\eta_{r k}$ to be 1 and 0 otherwise.

Subproblem 2 (related to decision variables $h_{r b}^{\mathrm{dir}}$ ). One has

$$
\begin{aligned}
& Z_{\text {sub3.2 }}\left(\mu_{1}, \mu_{15}, \mu_{22}\right) \\
& \quad=\min \left\{\sum_{r \in R} \sum_{b \in B \text { dir } \in \text { DIR }} \sum_{r b} h_{\text {dir }}\left[\mu_{r \text { dir }}^{1}+\sum_{n \in N}\left(\mu_{n r b \operatorname{dir}}^{22}-\mu_{n r b \operatorname{dir}}^{15}\right)\right]\right\},
\end{aligned}
$$

subject to

$$
\begin{gathered}
\sum_{b \in B} h_{r b}^{\mathrm{dir}} \leq 1 \quad \forall r \in R, \operatorname{dir} \in \mathrm{DIR}, \\
h_{r b}^{\mathrm{dir}}=0 \text { or } 1 \quad \forall r \in R, \\
b \in B, \quad \operatorname{dir} \in \mathrm{DIR} .
\end{gathered}
$$

Equation (Sub 3.2) can be further decomposed into $|R| \times$ |DIR| subproblems. For each RS $r$ and direction dir,

$$
\min \left\{\sum_{b \in B} h_{r b}^{\mathrm{dir}}\left[\mu_{r \mathrm{dir}}^{1}+\sum_{n \in N}\left(\mu_{n r b \mathrm{dir}}^{22}-\mu_{n r b \mathrm{dir}}^{15}\right)\right]\right\}
$$

Subject to (A.4a) and (A.4b).

For each (Sub 3.2.1), find the BS $b$ which can result in the smallest coefficient $\mu_{r \text { dir }}^{1}+\sum_{n \in N}\left(\mu_{n r b \operatorname{dir}}^{22}-\mu_{n r b \operatorname{dir}}^{15}\right)$ of $h_{r b}^{\mathrm{dir}}$; if the coefficient is negative, then set $h_{r b}^{\mathrm{dir}}$ to be 1 and 0 otherwise.

Subproblem 3 (related to decision variables $\kappa_{n s}^{\mathrm{dir}}, \pi_{s n}^{\mathrm{dir}}$ ). One has

$$
\begin{array}{r}
Z_{\text {sub3.2 }}\left(\mu_{3}, \mu_{4}, \mu_{5}, \mu_{6}, \mu_{7}, \mu_{8}, \mu_{9}, \mu_{14}, \mu_{15}, \mu_{16}\right) \\
=\min \sum_{n \in N}\left\{\sum _ { s \in B } \left[\sum_{\operatorname{dir} \in \operatorname{DIR}} \mu_{s}^{3} \kappa_{n s}^{\mathrm{dir}} \theta_{n}^{\mathrm{dir}}\right.\right. \\
+\mu_{n s}^{5}\left(\kappa_{n s}^{1}\left(P_{\min }^{N}-\pi_{s n}^{1}\right)\right) \\
+\mu_{n s}^{6}\left(\kappa_{n s}^{2}\left(P_{\min }^{R}-\pi_{n s}^{2}\right)\right) \\
\left.+\left(\mu_{n s}^{8} \pi_{s n}^{1}+\mu_{n s}^{9} \pi_{n s}^{2}\right)\right] \\
+\sum_{s \in R}\left[\sum _ { \operatorname { d i r } \in \mathrm { DIR } } \left(\mu_{n s \mathrm{dir}}^{4} \kappa_{n s}^{\mathrm{dir}}-\mu_{n s \mathrm{dir}}^{15} \kappa_{n s}^{\mathrm{dir}}\right.\right. \\
\left.+\sum_{i \in R} \sum_{b \in B} \mu_{n i s b \operatorname{dir}}^{16} \kappa_{n s}^{\mathrm{dir}} \bar{M}\right)
\end{array}
$$

$$
\begin{gathered}
+\mu_{n s}^{5}\left(\kappa_{n s}^{1}\left(\bar{M}-\pi_{s n}^{1}\right)\right) \\
+\mu_{n s}^{6}\left(\kappa_{n s}^{2}\left(\bar{M}-\pi_{n s}^{2}\right)\right) \\
\left.+\left(\kappa_{n s}^{1} \mu_{n s}^{8} \pi_{s n}^{1}+\kappa_{n s}^{2} \mu_{n s}^{9} \pi_{n s}^{2}\right)\right] \\
+\sum_{s \in\{R \cup B\}}\left[\mu_{n}^{7} \kappa_{n s}^{1}\left(\pi_{s n}^{1}\right)\right. \\
\left.\left.+\mu_{n}^{14} \kappa_{n s}^{2} \operatorname{BEP}\left(\pi_{n s}^{2}\right)\right]\right\}
\end{gathered}
$$

(Sub 3.3)

subject to

$$
\begin{gathered}
\sum_{b \in B} \kappa_{n b}^{\mathrm{dir}}+\kappa_{n r}^{\mathrm{dir}} \leq 1 \quad \forall n \in N, \\
r \in R, \quad \operatorname{dir} \in \mathrm{DIR}, \\
1 \leq \sum_{s \in\{R \cup B\}} \kappa_{n s}^{\mathrm{dir}} \quad \forall n \in N, \operatorname{dir} \in \mathrm{DIR}, \\
\sum_{r \in R} \kappa_{n r}^{\mathrm{dir}} \leq \mathrm{SD}^{\mathrm{dir}} \quad \forall n \in N, \operatorname{dir} \in \mathrm{DIR}, \\
0 \leq \pi_{s n}^{\mathrm{dir}} \leq \bar{\pi} \quad \forall n \in N, \\
s \in\{R \cup B\}, \quad \operatorname{dir} \in \mathrm{DIR}, \\
\kappa_{n s}^{\mathrm{dir}}=0 \text { or } 1 \quad \forall n \in N, \\
s \in\{R \cup B\}, \quad \operatorname{dir} \in \mathrm{DIR} .
\end{gathered}
$$

Equation (Sub 3.3) can be further decomposed into $|N|$ independent subproblems. For each MC $n$,

$$
\begin{aligned}
\min \left\{\sum _ { s \in B } \left[\sum_{\mathrm{dir} \in \mathrm{DIR}} \mu_{s}^{3} \kappa_{n s}^{\mathrm{dir}} \theta_{n}^{\mathrm{dir}}\right.\right. & \\
& +\mu_{n s}^{5}\left(\kappa_{n s}^{1}\left(P_{\min }^{N}-\pi_{s n}^{1}\right)\right) \\
& +\mu_{n s}^{6}\left(\kappa_{n s}^{2}\left(P_{\min }^{R}-\pi_{n s}^{2}\right)\right) \\
& \left.+\left(\mu_{n s}^{8} \pi_{s n}^{1}+\mu_{n s}^{9} \pi_{n s}^{2}\right)\right] \\
+ & \sum_{s \in R}\left[\sum _ { \operatorname { d i r } \in \mathrm { DIR } } \left(\mu_{n s \mathrm{dir}}^{4} \mathrm{c}_{n s}^{\mathrm{dir}}-\mu_{n s \mathrm{dir}}^{15} \kappa_{n s}^{\mathrm{dir}}\right.\right. \\
& \left.+\sum_{i \in R} \sum_{b \in B} \mu_{n i s b \mathrm{dir}}^{16} \kappa_{n s}^{\mathrm{dir}} \bar{M}\right) \\
& +\mu_{n s}^{6}\left(\kappa_{n s}^{1}\left(\kappa_{n s}^{2}\left(\bar{M}-\pi_{s n}^{1}\right)\right)\right. \\
& \left.+\left(\kappa_{n s}^{1} \mu_{n s}^{8} \pi_{s n}^{1}+\kappa_{n s}^{2}\right)\right)
\end{aligned}
$$




$$
\begin{aligned}
+\sum_{n \in N} \sum_{s \in\{R \cup B\}}\left[\mu_{n}^{7} \kappa_{n s}^{1}\left(\pi_{s n}^{1}\right)\right. & \\
\left.+\mu_{n}^{14} \kappa_{n s}^{2} \operatorname{BEP}\left(\pi_{n s}^{2}\right)\right] & \}
\end{aligned}
$$

(Sub 3.3.1)

subject to (A.5a), (A.5b), (A.5c), (A.5d), and (A.5e).

The two directions of DL and UL are independent; (Sub 3.3.1) can be decomposed into DL and UL subproblems. Constraint (A.5a) illustrates that once an MC homes to exactly a BS, it cannot home to RS anymore, and vice versa, so (Sub 3.3.1) can be rewritten into following forms. In both directions, while MC $n$ homes to a BS $b$, the decision variable $\kappa_{n b}^{\text {dir }}=1$. For each MC $n$

$$
\begin{gathered}
\left(\operatorname { m i n } \left\{\sum _ { b \in B } \left[\mu_{b}^{3} \theta_{n}^{1}+P_{\min }^{N} \mu_{n b}^{5}\right.\right.\right. \\
\left.-\pi_{b n}^{1}\left(\mu_{n b}^{5}+\mu_{n}^{7}-\mu_{n s}^{8}\right)\right] \kappa_{n b}^{1}, \\
\sum_{r \in R}\left[\mu_{n r 1}^{4}+\sum_{b \in B} \mu_{n r b 1}^{15}\right. \\
+P_{\min }^{N} \mu_{n r}^{5}+\bar{M} \sum_{i \in R} \sum_{b \in B} \mu_{n i r b 1}^{16} \\
\left.-\pi_{r n}^{1}\left(\mu_{n r}^{5}+\mu_{n}^{7}-\mu_{n r}^{8}\right)\right] \\
\left.\left.\times \kappa_{n r}^{1}\right\}\right) \quad(\text { for DL }) \\
+\left(\operatorname { m i n } \left\{\sum _ { b \in B } \left[\mu_{b}^{3} \theta_{n}^{2}+P_{\min }^{R} \mu_{n b}^{6}\right.\right.\right. \\
-\pi_{n b}^{2}\left(\mu_{n b}^{6}-\mu_{n s}^{9}\right) \\
\left.+\mu_{n}^{14} \mathrm{BEP}\left(\pi_{n b}^{2}\right)\right] \kappa_{n b}^{2},
\end{gathered}
$$

$$
\begin{aligned}
\sum_{r \in R}\left[\mu_{n r 2}^{4}\right. & +\sum_{b \in B} \mu_{n r b 2}^{15} \\
& +P_{\min }^{R} \mu_{n r}^{6}+\bar{M} \sum_{i \in R} \sum_{b \in B} \mu_{n i r b 2}^{16} \\
& -\pi_{n r}^{2}\left(\mu_{n r}^{6}-\mu_{n r}^{9}\right) \\
& \left.\left.\left.+\mu_{n}^{14} \operatorname{BEP}\left(\pi_{n r}^{2}\right)\right] \kappa_{n r}^{2}\right\}\right) \quad \text { (for UL) }
\end{aligned}
$$

(Sub 3.3.2)

subject to (A.5a), (A.5b), (A.5c), (A.5d), and (A.5e).

The algorithm to optimally solve (Sub 3.3.2) is illustrated in the following.

For $D L$

Step 1. Use SNR function to calculate the SNR value $\pi_{b n}^{1}$ from every BS to MC $n$.

Step 2. Find the BS $b$ which can result in the smallest coefficient $\mu_{b}^{3} \theta_{n}^{1}+P_{\min }^{N} \mu_{n b}^{5}-\pi_{b n}^{1}\left(\mu_{n b}^{5}+\mu_{n}^{7}-\mu_{n s}^{8}\right)$ of $\kappa_{n b}^{1}$.

Step 3. Examining all sets of configuration for each RS in the SNR function to determine the SNR value $\pi_{r n}^{1}$; meanwhile, to find the RSs which can result in the first $\mathrm{SD}^{1}$ smallest coefficient $\mu_{n s 1}^{4}+\sum_{b \in B} \mu_{n s b 1}^{15}+P_{\min }^{N} \mu_{n s}^{5}+\bar{M} \sum_{i \in R} \sum_{b \in B} \mu_{n i s b 1}^{16}-$ $\pi_{s n}^{1}\left(\mu_{n s}^{5}+\mu_{n}^{7}-\mu_{n s}^{8}\right)$ of $\kappa_{n r}^{1}$. The summation of these $\mathrm{SD}^{1}$ amount of coefficients can be taken into consideration excluding positive ones bigger than the smallest coefficient for the further calculations. That is, we at least had the smallest coefficient for further steps, whether it is negative or not.

Step 4. If the coefficient of $\kappa_{n b}^{1}$ in Step 2 is smaller than the summation of coefficient of $\mathrm{SD}^{1}$ smallest coefficient of $\kappa_{n r}^{1}$ in Step 3 , then set $\kappa_{n b}^{1}$ to be 1 ; otherwise, set these $\mathrm{SD}^{1}$ of $\kappa_{n r}^{1}$ to be 1 .

For UL. Repeat Step 1 to Step 4 to determine $\kappa_{n s}^{2}$ and $\pi_{s n}^{2}$ with spatial diversity number $\mathrm{SD}^{2}$.

Subproblem 4 (related to decision variable $x_{n r p}^{\mathrm{dir}}$ ). One has

$$
\begin{aligned}
& Z_{\text {sub }}\left(\mu_{16}, \mu_{19}, \mu_{22}\right) \\
& \quad=\min \left\{\sum_{n \in N} \sum_{r \in R} \sum_{\text {dir } \in \text { DIR }} \sum_{b \in B} \sum_{p \in P_{b r}}\left[\sum_{u \in\{R \cup B\}} \sum_{v \in\{R \cup B\}} \delta_{p u v}\left(\mu_{n r u v \text { dir }}^{19}+\sum_{j \in R} \mu_{n r j b d i r}^{16}-\sum_{i \in R} \mu_{n i r b d i r}^{16}\right)-\mu_{n r b \operatorname{dir}}^{22}\right] x_{n r p}^{\text {dir }}\right\}
\end{aligned}
$$

subject to

$$
\begin{array}{r}
\sum_{b \in B} \sum_{p \in P_{b r}} x_{n r p}^{\mathrm{dir}} \leq 1 \quad \forall n \in N, \\
r \in R, \quad \operatorname{dir} \in \mathrm{DIR},
\end{array}
$$

$$
\begin{gathered}
x_{n r p}^{\mathrm{dir}}=0 \text { or } 1 \quad \forall n \in N, r \in R, \\
p \in P_{b r}, \quad b \in B, \quad \operatorname{dir} \in \mathrm{DIR} .
\end{gathered}
$$

Equation (Sub 3.4) can be further decomposed into $|N| \times$ $|R| \times \mid$ DIR $\mid$ independent shortest path problems with arc weight $\mu_{n r u v \text { dir }}^{19}+\sum_{j \in R} \mu_{n r j b d i r}^{16}-\sum_{i \in R} \mu_{n i r b d i r}^{16}$. For each shortest 
path problem, it can be effectively solved by Bellman Ford's minimum cost shortest path algorithm. For each RS $n$, RS $r$, DIR dir, if the total cost of the shortest path is smaller than $\mu_{n r b \operatorname{dir}}^{22}$, then set $x_{n r p}^{\mathrm{dir}}$ to be 1 and 0 otherwise.

Subproblem 5 (related to decision variables $\left.y_{n u v}^{\mathrm{dir}}, \phi_{u v}^{\mathrm{dir}}\right)$. One has

$$
\begin{aligned}
& Z_{\text {sub }}\left(\mu_{2}, \mu_{3}, \mu_{10}, \mu_{11}, \mu_{12}, \mu_{13}, \mu_{17}, \mu_{18}, \mu_{19}, \mu_{21}\right) \\
& =\min \left\{\sum_{n \in N}\left[\sum_{u \in\{R \cup B\}} \sum_{v \in R} \mu_{v}^{2}+\sum_{u \in B} \sum_{v \in R} \mu_{u}^{3}\right] y_{n u v}^{1} \theta_{n}^{1}\right. \\
& +\sum_{n \in N}\left[\sum_{u \in R} \sum_{v \in\{R \cup B\}} \mu_{u}^{2}+\sum_{u \in R} \sum_{v \in B} \mu_{v}^{3}\right] y_{n u v}^{2} \theta_{n}^{2} \\
& -\sum_{n \in N} \sum_{v \in\{R \cup B\}} \sum_{u \in\{R \cup B\}} \mu_{n v}^{11} y_{n u v}^{2} \phi_{u v}^{2} \\
& +\sum_{n \in N} \sum_{u \in\{R \cup B\}} \sum_{v \in\{R \cup B\}} \mu_{n}^{13} y_{n u v}^{1} \operatorname{BEP}\left(\phi_{u v}^{1}\right) \\
& +\sum_{n \in N} \sum_{u \in\{R \cup B\}} \sum_{v \in\{R \cup B\}}\left[\mu_{n u v}^{17} y_{n u v}^{1}+\mu_{n u v}^{18} y_{n u v}^{2}\right] \\
& +\sum_{u \in\{R \cup B\}} \sum_{v \in\{R \cup B\}} \sum_{\operatorname{dir} \in \mathrm{DIR}}\left[\sum _ { n \in N } \left(\mu_{n u v \operatorname{dir}}^{10}\left(\bar{M}-\phi_{u v}^{\mathrm{dir}}\right)\right.\right. \\
& +\mu_{u v \operatorname{dir}}^{21} \theta_{n}^{\mathrm{dir}} \\
& \left.\left.-\sum_{r \in R} \mu_{n r u v \operatorname{dir}}^{19}\right) y_{n u v}^{\mathrm{dir}}\right] \\
& +\sum_{n \in N} \sum_{u \in\{R \cup B\}} \sum_{v \in\{R \cup B\}} \sum_{\operatorname{dir} \in \mathrm{DIR}}\left[\mu_{n u v \mathrm{dir}}^{12} \phi_{u v}^{\mathrm{dir}}+\bar{M}\right] y_{n u v}^{\mathrm{dir}} \\
& \left.-\sum_{u \in\{R \cup B\}} \sum_{v \in\{R \cup B\}} \sum_{\operatorname{dir} \in \mathrm{DIR}} \mu_{u v \operatorname{dir}}^{21} C_{u v}\left(\phi_{u v}^{\mathrm{dir}}\right)\right\},
\end{aligned}
$$

(Sub 3.5)

subject to

$$
\begin{gathered}
0 \leq \phi_{u v}^{\mathrm{dir}} \leq \bar{\phi} \quad \forall u, v \in\{R \cup B\}, \operatorname{dir} \in \mathrm{DIR}, \\
y_{n u v}^{\mathrm{dir}}=0 \text { or } 1 \quad \forall n \in N, \\
u, v \in\{R \cup B\}, \quad \operatorname{dir} \in \mathrm{DIR} .
\end{gathered}
$$

Similar to (Sub 3.3), Since DL and UL are two independent transmission directions, we can rewrite (Sub 3.5) into DL and UL subproblems:

$$
\begin{aligned}
\min \left\{\sum _ { u \in \{ R \cup B \} } \sum _ { v \in \{ R \cup B \} } \left[\sum_{n \in N}[\right.\right. & \mu_{n u v 1}^{10} P_{\min }^{R} \\
& +\left(\mu_{u v 1}^{12}-\mu_{n u v 1}^{10}\right) \phi_{u v}^{1} \theta_{n}^{1}
\end{aligned}
$$

$$
\begin{aligned}
& +\mu_{n u v}^{17}-\sum_{r \in R} \mu_{n r u v 1}^{19} \\
& +\left(\mu_{v}^{2}+\mu_{u}^{3}+\mu_{u v 1}^{21}\right) \\
& \left.+\mu_{n}^{13} \operatorname{BEP}\left(\phi_{u v}^{1}\right)\right] y_{n u v}^{1} \\
& \left.\left.-\mu_{u v 1}^{21} C_{u v}\left(\phi_{u v}^{1}\right)\right]\right\} \quad(\text { for } \mathrm{DL}) \\
& +\min \left\{\sum _ { u \in \{ R \cup B \} } \sum _ { v \in \{ R \cup B \} } \left[\sum _ { n \in N } \left[\mu_{n u v 2}^{10} P_{\min }^{R}\right.\right.\right. \\
& +\left(\mu_{u v 2}^{12}-\mu_{n u v 2}^{10}\right) \phi_{u v}^{2} \\
& +\mu_{n u v}^{18}-\sum_{r \in R} \mu_{n r u v 2}^{19} \\
& +\left(\mu_{u}^{2}+\mu_{v}^{3}+\mu_{u v 2}^{21}\right) \theta_{n}^{2} \\
& \left.-\mu_{n}^{11} \phi_{u v}^{2}\right] y_{n u v}^{2} \\
& \left.\left.-\mu_{u v 2}^{21} C_{u v}\left(\phi_{u v}^{2}\right)\right]\right\} \quad(\text { for DL), }
\end{aligned}
$$

(Sub 3.5.1)

subject to (A.7a) and (A.7b).

Equation (Sub 3.5.1) can then be decomposed into $\mid R \cup$ $B|\times| R \cup B \mid$ independent subproblems. For each link $u v$,

$$
\begin{gathered}
\min \left\{\sum _ { n \in N } \left[\mu_{n u v 1}^{10} P_{\min }^{R}+\left(\mu_{u v 1}^{12}-\mu_{n u v 1}^{10}\right) \phi_{u v}^{1}\right.\right. \\
+\mu_{n u v}^{17}-\sum_{r \in R} \mu_{n r u v 1}^{19} \\
+\left(\mu_{v}^{2}+\mu_{u}^{3}+\mu_{u v 1}^{21}\right) \theta_{n}^{1} \\
\left.+\mu_{n}^{13} \operatorname{BEP}\left(\phi_{u v}^{1}\right)\right] y_{n u v}^{1} \\
\left.-\mu_{u v 1}^{21} C_{u v}\left(\phi_{u v}^{1}\right)\right\} \text { (for DL) }
\end{gathered}
$$

$$
\begin{aligned}
+\min \left\{\sum _ { n \in N } \left[\mu_{n u v 2}^{10} P_{\min }^{R}\right.\right. & \\
& +\left(\mu_{u v 2}^{12}-\mu_{n u v 2}^{10}\right) \phi_{u v}^{2} \\
& +\mu_{n u v}^{18}-\sum_{r \in R} \mu_{n r u v 2}^{19} \\
& +\left(\mu_{u}^{2}+\mu_{v}^{3}+\mu_{u v 2}^{21}\right) \theta_{n}^{2} \\
& \left.-\mu_{n}^{11} \phi_{u v}^{2}\right] y_{n u v}^{2} \\
- & \left.\mu_{u v 2}^{21} C_{u v}\left(\phi_{u v}^{2}\right)\right\} \quad(\text { for } \mathrm{DL}),
\end{aligned}
$$

(Sub 3.5.2)

subject to (A.7a) and (A.7b). 
The algorithm to optimally solve (Sub 3.5.2) is illustrated in the following.

For DL

Step 1. Examining all sets of configuration for each source node $u$ in the SNR function to determine the SNR value $\phi_{u v}^{1}$ which can result in the smallest summation of coefficient $\sum_{n \in N}\left[\mu_{n u v 1}^{10} P_{\min }^{R}+\left(\mu_{u v 1}^{12}-\mu_{n u v 1}^{10}\right) \phi_{u v}^{1}+\mu_{n u v}^{17}-\sum_{r \in R} \mu_{n r u v 1}^{19}+\left(\mu_{v}^{2}+\right.\right.$ $\left.\left.\mu_{u}^{3}+\mu_{u v 1}^{21}\right) \theta_{n}^{1}+\mu_{n}^{13} \operatorname{BEP}\left(\phi_{u v}^{1}\right)\right]$ of $y_{n u v}^{1}$. If all individual coefficients are positive, then set $\phi_{u v}^{1}$ and all $y_{n u v}^{1}$ to be 0 .

Step 2. For each MC $n$, if the coefficient $\mu_{n u v 1}^{10} P_{\min }^{R}+\left(\mu_{u v 1}^{12}-\right.$ $\left.\mu_{n u v 1}^{10}\right) \phi_{u v}^{1}+\mu_{n u v}^{17}-\sum_{r \in R} \mu_{n r u v 1}^{19}+\left(\mu_{v}^{2}+\mu_{u}^{3}+\mu_{u v 1}^{21}\right) \theta_{n}^{1}+\mu_{n}^{13} \operatorname{BEP}\left(\phi_{u v}^{1}\right)$ is negative, then set $y_{n u v}^{1}$ to be 1 and 0 otherwise.

For UL. Repeat Steps 1 and 2 to determine $\phi_{u v}^{2}$ and $y_{n u v}^{2}$.

Subproblem 6 (related to decision variable $\omega_{n}$ ). One has

$$
\begin{aligned}
& Z_{\text {sub }}\left(\mu_{7}, \mu_{13}\right) \\
& =\min \left\{\sum_{n \in N}\left[\mu_{n}^{13} \operatorname{BER}\left(\omega_{n}\right)+\mu_{n}^{7} \omega_{n}\right]\right\},
\end{aligned}
$$

subject to

$$
\begin{gathered}
\omega_{n}^{\min } \leq \omega_{n} \leq \omega_{n}^{\max } \quad \forall n \in N, \\
\omega_{n} \in \Omega_{n}=\{0, \Delta, 2 \Delta, 3 \Delta, 4 \Delta, \ldots\} \quad \forall n \in N .
\end{gathered}
$$

For (Sub 3.6) can be solvable, we introduced constraint (A.8b) into this subproblem to transform $\omega_{n}$ from continuous to discrete. Then, (Sub 3.6) can be further decomposed into $|N|$ independent subproblems. For each $\mathrm{MC} n$,

$$
\min \left\{\mu_{n}^{13} \operatorname{BER}\left(\omega_{n}\right)+\mu_{n}^{7} \omega_{n}\right\},
$$

subject to (A.8a) and (A.8b).

We can calculate the value of (Sub 3.6.1) by examining every $\omega_{n}$ exhaustively. Set $\omega_{n}$ while it can result in the smallest value of (Sub 3.6.1). Here, we applied the interval $\Delta$ to be 0.01 .

Subproblem 7 (related to decision variable $\varepsilon_{n v}$ ). One has

$$
\begin{aligned}
& Z_{\text {sub }}\left(\mu_{11}, \mu_{14}\right) \\
& \quad=\min \left\{\sum_{n \in N} \sum_{v \in\{R \cup B\}}\left[\mu_{n}^{14} \operatorname{BER}\left(\varepsilon_{n v}\right)+\mu_{n v}^{11} \varepsilon_{n v}\right]\right\},
\end{aligned}
$$

subject to

$$
\begin{aligned}
\varepsilon_{n v}^{\min } & \leq \varepsilon_{n v} \leq \varepsilon_{n v}^{\max } \quad \forall n \in N, v \in\{R \cup B\}, \quad \text { (A.9a) } \\
\varepsilon_{n v} \in E_{n v} & =\{0, \Delta, 2 \Delta, 3 \Delta, 4 \Delta, \ldots\} \quad \forall n \in N, v \in\{R \cup B\} .
\end{aligned}
$$

In the same situation like Subproblem 7, we introduced constraint (A.9b) into subproblem 7 to transform $\varepsilon_{n v}$ from continuous to discrete. Equation (Sub 3.7) can be further decomposed into $|N| \times|R \cup B|$ independent subproblems. For each MC $n$, RS (or BS) $v$,

$$
\min \left\{\mu_{n}^{14} \operatorname{BER}\left(\varepsilon_{n v}\right)+\mu_{n v}^{11} \varepsilon_{n v}\right\}
$$

subject to (A.9a), and (A.9b).

Similar to (Sub 3.6.1), (Sub 3.7.1) can be solved by exhaustively examining $\varepsilon_{n v}$ to find out the smallest value of this problem; then set $\varepsilon_{n v}$. Here, we applied the interval $\Delta$ to be 0.01 .

The Dual Problem and the Subgradient Method. According to the algorithms proposed previously, the Lagrangian relaxation problem can be solved effectively and optimally. Based on the weak Lagrangian duality theorem, the objective value of $Z_{D}\left(\mu_{1}, \mu_{2}, \mu_{3}, \mu_{4}, \mu_{5}, \mu_{6}, \mu_{7}, \mu_{8}, \mu_{9}, \mu_{10}, \mu_{11}, \mu_{12}, \mu_{13}\right.$, $\left.\mu_{14}, \mu_{15}, \mu_{16}, \mu_{17}, \mu_{18}, \mu_{19}, \mu_{20}, \mu_{21}, \mu_{22}\right)$ is a lower bound of $Z_{I P}$. The following dual problem is constructed to calculate the tightest lower bound and solved the dual problem by using the subgradient method.

Dual Problem (D). One has

$$
\begin{gathered}
Z_{D}=\max Z_{D}\left(\mu_{1}, \mu_{2}, \mu_{3}, \mu_{4}, \mu_{5}, \mu_{6},\right. \\
\mu_{7}, \mu_{8}, \mu_{9}, \mu_{10}, \mu_{11}, \\
\mu_{12}, \mu_{13}, \mu_{14}, \mu_{15}, \mu_{16}, \\
\left.\mu_{17}, \mu_{18}, \mu_{19}, \mu_{20}, \mu_{21}, \mu_{22}\right),
\end{gathered}
$$

subject to

$$
\begin{aligned}
& \mu_{1}, \mu_{2}, \mu_{3}, \mu_{4}, \mu_{5}, \mu_{6}, \mu_{7}, \mu_{8}, \mu_{9}, \\
& \mu_{10}, \mu_{11}, \mu_{12}, \mu_{13}, \mu_{14}, \mu_{15}, \\
& \mu_{16}, \mu_{17}, \mu_{18}, \mu_{19}, \mu_{20}, \mu_{21}, \mu_{22} \geq 0 .
\end{aligned}
$$

Let the vector $S$ be a subgradient of $Z_{D}=$ $\max Z_{D}\left(\mu_{1}, \mu_{2}, \mu_{3}, \mu_{4}, \mu_{5}, \mu_{6}, \mu_{7}, \mu_{8}, \mu_{9}, \mu_{10}, \mu_{11}, \mu_{12}, \mu_{13}, \mu_{14}\right.$, $\left.\mu_{15}, \mu_{16}, \mu_{17}, \mu_{18}, \mu_{19}, \mu_{20}, \mu_{21}, \mu_{22}\right)$. Then, in iteration $k$ of the subgradient procedure, the multiplier vector $m^{k}=$ $\left(\mu_{1}^{k}, \mu_{2}^{k}, \mu_{3}^{k}, \mu_{4}^{k}, \mu_{5}^{k}, \mu_{6}^{k}, \mu_{7}^{k}, \mu_{8}^{k}, \mu_{9}^{k}, \mu_{10}^{k}, \mu_{11}^{k}, \mu_{12}^{k}, \mu_{13}^{k}, \mu_{14}^{k}, \mu_{15}^{k}, \mu_{16}^{k}\right.$, $\left.\mu_{17}^{k}, \mu_{18}^{k}, \mu_{19}^{k}, \mu_{20}^{k}, \mu_{21}^{k}, \mu_{22}^{k}\right)$ is updated by $m^{k+1}=m^{k}+t^{k} S^{k}$. The step size $t^{k}$ is determined by $t^{k}=\lambda\left(\left(Z_{I P}^{*}-Z_{D}\left(m^{k}\right)\right) /\left\|S^{k}\right\|^{2}\right)$. $Z_{I P}^{*}$ is the best primal objective function value found by iteration $k . \lambda$ is a constant where $0 \leq \lambda \leq 2$.

\section{Conflict of Interests}

It is hereby asserted that all the coauthors of this paper do not have any personal or financial interest with any model or system used in this paper.

\section{References}

[1] E. Fox, "North American ARPU growth outpaces the world: a look at wireless forecast drivers," Yankee Group, March 2006. 
[2] D. Wang, J. Li, K. Xing, S. Jin, and K. Liu, "Real-time, reallocation, and real-identity service information (R3SI) based application enabled mobile service architecture in cellular networks," in Proceedings of the 32nd International Conference on Distributed Computing Systems Workshops (ICDCSW '12), pp. 315-323, Macau.

[3] A. Engels, M. Reyer, and R. Mathar, "Profit-oriented combination of multiple objectives for planning and configuration of 4G multi-hop relay networks," in Proceedings of the 7th International Symposium on Wireless Communication Systems (ISWCS '10), pp. 330-334, September 2010.

[4] 3GPP, http://www.3gpp.org/.

[5] “3GPP TR 36. 814," Evolved Universal Terrestrial Radio Access (E-UTRA), Further Advancements for E-UTRA Physical Layer Aspects.

[6] “3GPP TR 36. 806," Evolved Universal Terrestrial Radio Access (E-UTRA), Relay Architectures for E-UTRA (LTE-Advanced).

[7] “3GPP R3-093149," Comprehensive Comparison among Type-I Relay Alternatives, 2009.

[8] “3GPP R1-101825,” Backhaul UL Subframe Allocation in TDD LTE-A Relay, 2010.

[9] Y. Yang, H. Hu, J. Xu, and G. Mao, "Relay technologies for WiMAX and LTE-Advanced mobile systems," IEEE Communications Magazine, vol. 47, no. 10, pp. 100-105, 2009.

[10] S. W. Peters, A. Y. Panah, K. T. Truong, and R. W. Heath, "Relay architectures for 3GPP LTE-advanced," Eurasip Journal on Wireless Communications and Networking, vol. 2009, Article ID 618787, 2009.

[11] X. Wang, S.-J. Horng, R.-G. Cheng, and P. Fan, "Call dropping performance analysis of the eNB-first channel access policy in LTE-Advanced relay networks," in Proceedings of the IEEE 7th International Conference on Wireless and Mobile Computing, Networking and Communications (WiMob '11), pp. 43-50, October 2011.

[12] C. Huang, M. Zeng, and S. Cui, "Achievable rates of two-hop interference networks with conferencing relays," in Proceedings of the 54th Annual IEEE Global Telecommunications Conference: "Energizing Global Communications" (GLOBECOM '11), pp. 1-6, Houston, Tex, USA, December 2011.

[13] V. D. Meulen and C. Edward, Transmission of Information in a T-Terminal Discrete Memoryless Channel, Department of Statistics, University of California, Berkeley, Calif, USA, 1968.

[14] R. Pabst, B. H. Walke, D. C. Schultz et al., "Relay-based deployment concepts for wireless and mobile broadband radio," IEEE Communications Magazine, vol. 42, no. 9, pp. 80-89, 2004.

[15] H. Li, L. Liu, G. Li, Y. Kim, and J. Zhang, "Multicell cooperation and MIMO technologies for broadcasting and broadband communications," International Journal of Digital Multimedia Broadcasting, vol. 2010, Article ID 848527, 2 pages, 2010.

[16] K. Schober, R. Wichman, and T. Roman, "Layer arrangement for single-user coordinated multi-point transmission," in Proceedings of the 46th Annual Conference on Information Sciences and Systems (CISS '12), pp. 1-5.

[17] A. Sendonaris, E. Erkip, and B. Aazhang, "User cooperation diversity, Part I: system description," IEEE Transactions on Communications, vol. 51, no. 11, pp. 1927-1938, 2003.

[18] A. Sendonaris, E. Erkip, and B. Aazhang, "User cooperation diversity, Part II: implementation aspects and performance analysis," IEEE Transactions on Communications, vol. 51, no. 11, pp. 1939-1948, 2003.
[19] J. Yang, Z. Zhang, Z. Jiang, X. Jiang, and M. He, "Study on joint-coding based on pre-coding and STBC in coordinated multi-point system," in Proceedings of the Spring Congress on Engineering and Technology (S-CET '12), pp. 1-4.

[20] M. Rahman and H. Yanikomeroglu, "Interference avoidance through dynamic downlink OFDMA subchannel allocation using intercell coordination," in Proceedings of the IEEE 67th Vehicular Technology Conference-Spring (VTC '08), pp. 16301635, May 2008.

[21] M. Rahman, H. Yanikomeroglu, and W. Wong, "Interference avoidance with dynamic inter-cell coordination for downlink LTE system," in Proceedings of the IEEE Wireless Communications and Networking Conference (WCNC '09), pp. 1238-1243, April 2009.

[22] G. Amarasuriya, C. Tellambura, and M. Ardakani, "Performance analysis framework for transmit antenna selection strategies of cooperative MIMO AF relay networks," IEEE Transactions on Vehicular Technology, vol. 60, no. 7, pp. 30303044, 2011.

[23] A. B. Saleh, O. Bulakci, J. Hämäläinen, S. Redana, and B. Raaf, "Analysis of the impact of site planning on the performance of relay deployments," IEEE Transactions on Vehicular Technology, vol. 61, no. 7, pp. 3139-3150, 2012.

[24] N. Kong and L. B. Milstein, "Average SNR of a generalized diversity selection combining scheme," IEEE Communications Letters, vol. 3, no. 3, pp. 57-79, 1999.

[25] R. K. Mallik, M. Z. Win, J. W. Shao, M.-S. Alouini, and A. J. Goldsmith, "Channel capacity of adaptive transmission with maximal ratio combining in correlated Rayleigh fading," IEEE Transactions on Wireless Communications, vol. 3, no. 4, pp. 11241133, 2004.

[26] M. L. Fisher, "The Lagrangian relaxation method for solving integer programming problems," Management Science, vol. 27, no. 1, pp. 1-18, 1981.

[27] T. N. Lin, Content Delivery over Wireless Network- Radio Propagation: Issues \& Models, Department of Electrical Engineering, National Taiwan University, Taiwan.

[28] C. Langton, "Intuitive guide to principles of communications: all about modulation: Part II," 2002, http://complextoreal.com/.

[29] "Wireless Communications Laboratory," National Chung Cheng University, Taiwan. 


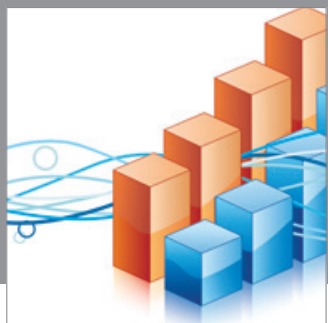

Advances in

Operations Research

mansans

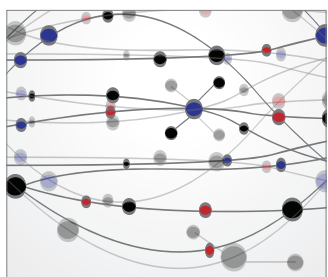

The Scientific World Journal
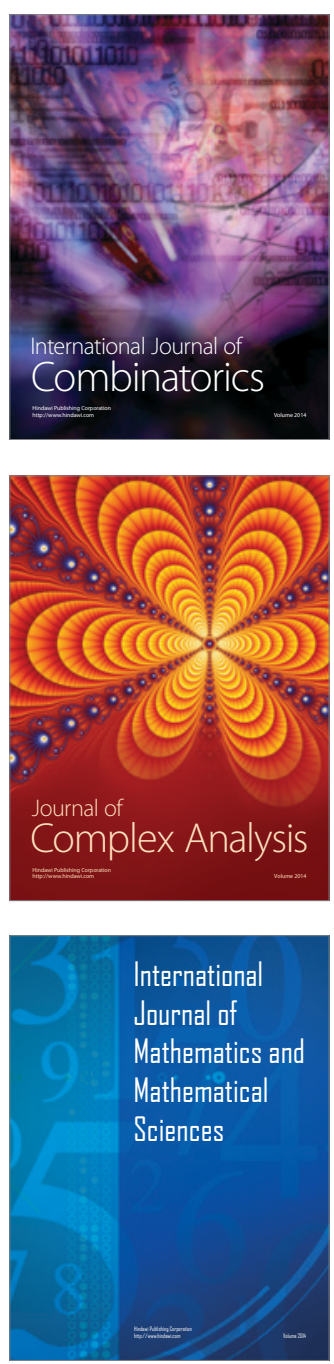
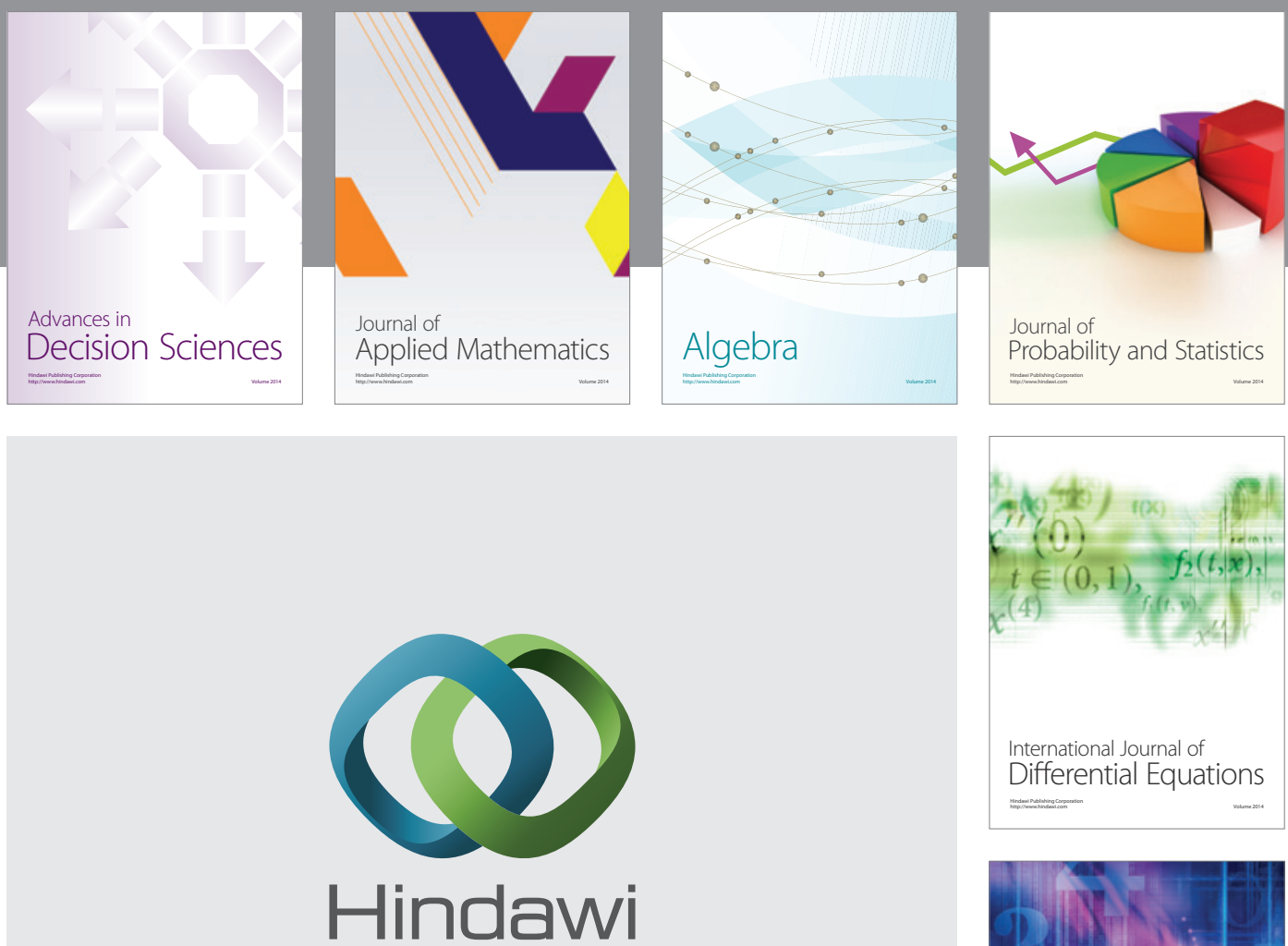

Submit your manuscripts at http://www.hindawi.com
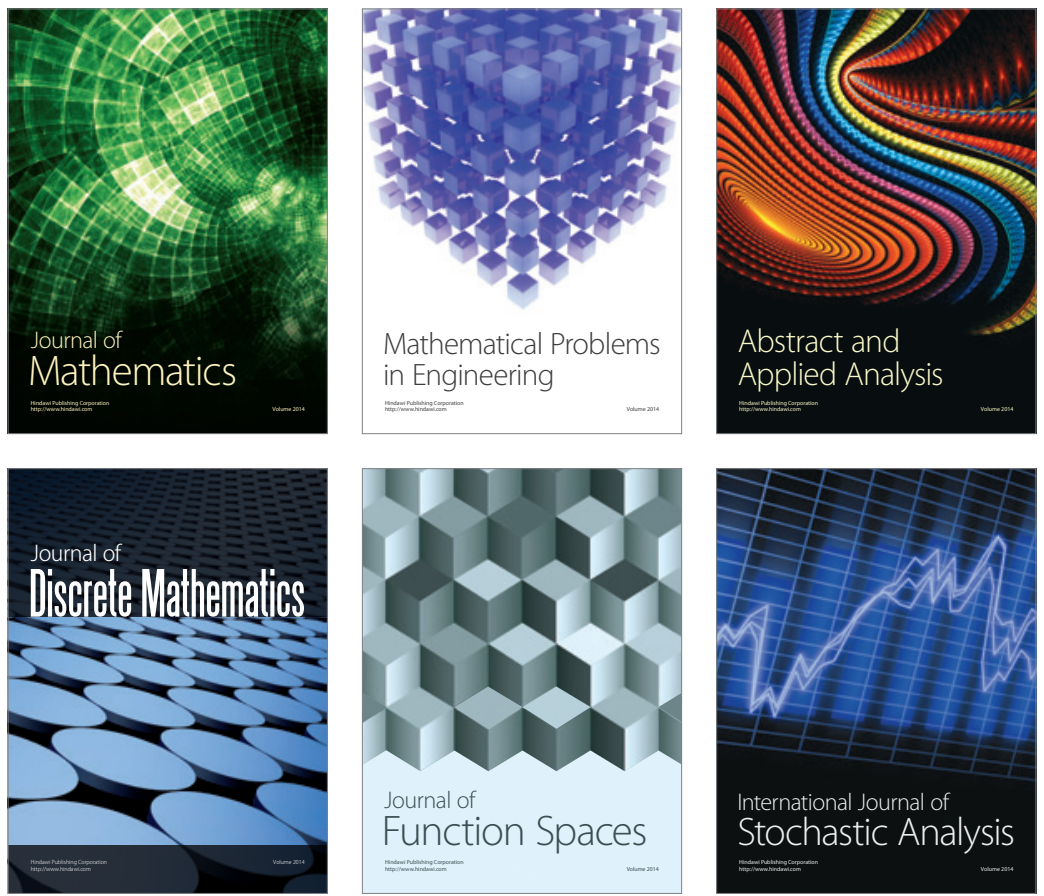

Journal of

Function Spaces

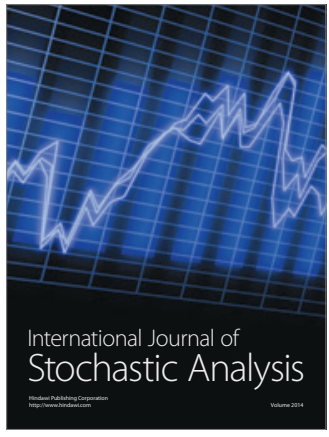

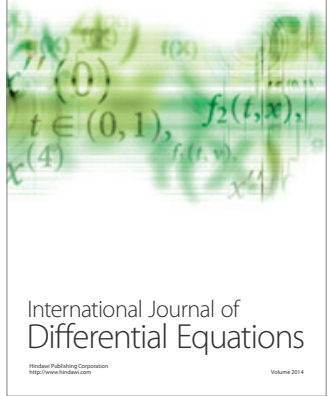
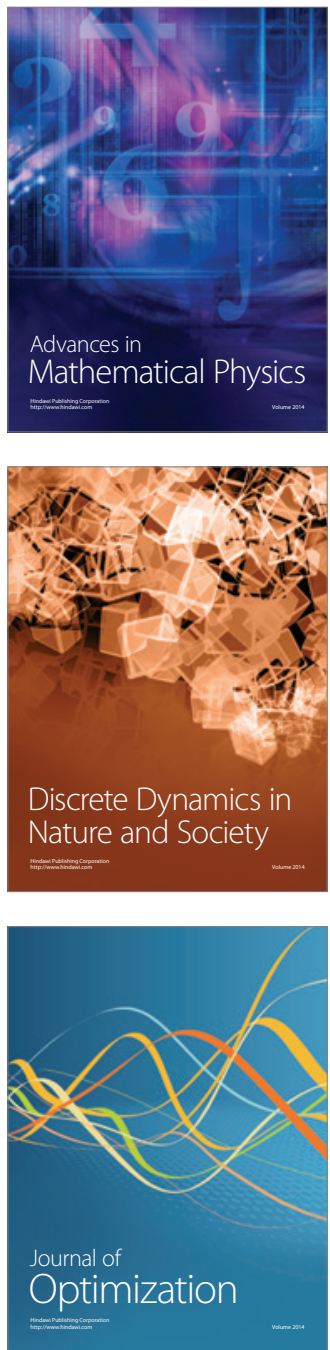